\title{
Morphological variation in Echinorhynchus truttae Schrank, 1788 and the $E$. bothniensis Zdzitowiecki \& Valtonen, 1987 species complex from freshwater fishes of northern Europe
}

\author{
Matthew T Wayland ${ }^{\dagger}$ \\ † Department of Zoology, University of Cambridge, Cambridge, United Kingdom
}

Corresponding author: Matthew T Wayland (mw283@cam.ac.uk)

Academic editor: Lyubomir Penev

Received: 01 Aug 2013 | Accepted: 27 Aug 2013 | Published: 16 Sep 2013

Citation: Wayland M (2013) Morphological variation in Echinorhynchus truttae Schrank, 1788 and the E. bothniensis Zdzitowiecki \& Valtonen, 1987 species complex from freshwater fishes of northern Europe. Biodiversity Data Journal 1: e975. doi: 10.3897/BDJ.1.e975

\section{Abstract}

Echinorhynchus truttae and the E. bothniensis species complex are common parasites of salmoniform and other fishes in northern Europe. E. bothniensis and its sibling species $E$. 'bothniensis' are thought to be closely related to the Nearctic E. leidyi Van Cleave, 1924 based on morphological similarity and common usage of a mysid intermediate host. This study provides the first analysis of morphological and meristic variation in $E$. truttae and expands our knowledge of anatomical variability in the E. bothniensis group. Morphological variability in E. truttae was found to be far greater than previously reported, with part of the variance attributable to sexual dimorphism. E. truttae, the two species of the E. bothniensis group and E. leidyi displayed considerable interspecific overlap in the ranges of all conventional morphological characters. However, Proboscis profiler, a tool for detecting acanthocephalan morphotypes using multivariate analysis of hook morphometrics, successfully separated $E$. truttae from the other taxa. The E. bothniensis species group could not be reliably distinguished from $E$. leidyi (or each other), providing further evidence of the affinity of these taxa. Observations on the distribution of $E$. truttae in its definitive host population are also reported. 


\section{Keywords}

Acanthocephala, Echinorhynchus truttae, Echinorhynchus bothniensis, Echinorhynchus leidyi, cryptic speciation, sibling species, morphology, morphometric, meristic, description, Proboscis Profiler, overdispersion, parasite, salmoniform

\section{Introduction}

Echinorhynchus bothniensis Zdzitowiecki \& Valtonen, 1987 was originally described from Osmerus eperlanus L. from the oligohaline waters of the Bothnian Bay, northern Baltic. In earlier studies (Timola 1980, Valtonen 1980, Valtonen 1983) this acanthocephalan had been determined as E. gadi Zoega in Müller, 1776 (see Zdzitowiecki and Valtonen 1987). The first evidence that $E$. 'gadi' in $O$. eperlanus was biologically distinct from $E$. gadi in Gadus morhua L. and other marine fish came from amphipod infection experiments; acanthors of $E$. gadi from Baltic G. morhua were infective to Gammarus zaddachi Sexton, but acanthors of E. 'gadi' from O. eperlanus were not (Valtonen et al. 1983). Moreover, Valtonen et al. (1983) noted that the mature females of $E$. 'gadi' from O. eperlanus were smaller than the mature females of $E$. gadi found in G. morhua. A detailed morphological study by Zdzitowiecki and Valtonen (1987) revealed marked differences in egg dimensions between $E$. 'gadi' from $O$. eperlanus and $E$. gadi from G. morhua. Furthermore, male $E$. 'gadi' from $O$. eperlanus tended to exhibit one or more pairs of parallel cement glands in contrast to the moniliform pattern displayed by E. gadi from G. morhua, although there was some interspecific overlap in cement gland arrangement. On the basis of these morphological differences Zdzitowiecki and Valtonen (1987) accorded specific rank to $E$. 'gadi' from $O$. eperlanus by naming it $E$. bothniensis. Other true definitive hosts (i.e. hosts in which gravid female worms have been found) of $E$. bothniensis from the Bothnian Bay include Lampetra fluviatilis (L.), Salmo trutta L., Lota lota (L.), Myoxocephalus quadricornis (L.) and Platichthys flesus (L.) (Valtonen and Crompton 1990). The intermediate hosts belongs to the Mysis relicta Lovén (Mysidacea) species group (Valtonen and Crompton 1990). It is important to note that this species group has recently been split, on the basis of molecular and morphological characters (Audzijonytè and Väinölä 2005) into four named taxa: M. relicta (sensu stricto), M. salemaai Audzijonyte \& Väinölä, 2005, M. segerstralei Audzijonyte \& Väinölä, 2005 and M. diluviana Audzijonyte \& Väinölä, 2005.

Using multilocus enzyme electrophoresis Väinölä et al. (1994) demonstrated that not only is $E$. bothniensis heterospecific to $E$. gadi, but that both taxa represent complexes of sibling species. One species of the $E$. bothniensis group was found in $O$. eperlanus from the Bothnian Bay and in O. eperlanus and M. relicta (sensu stricto) (as M. relicta sp. I sensu Väinölä 1986) from Lake Keitele, central Finland. Väinölä et al. (1994) referred to this species as $E$. bothniensis, since its range included the type-locality. A second species found in Coregonus lavaretus (L.), Platichthys flesus (L.), Salvelinus alpinus (L.) and Mysis segerstralei Audzijonyte \& Väinölä, 2005 (as M. relicta sp. III sensu Väinölä 1986) from 
Lake Pulmankijärvi, northern Finland was designated E. 'bothniensis' (Väinölä et al. 1994). Neither of the lacustrine populations of the E. bothniensis group have previously been studied morphologically.

E. bothniensis is morphologically very similar to the North American E. leidyi Van Cleave, 1924 (= Echinorhynchus salvelini Linkins in Ward \& Whipple, 1918 nec Schrank, 1788), but apparently differs slightly from the latter species in hook formula and cement gland arrangement (Zdzitowiecki and Valtonen 1987). Mysis relicta (sensu lato) is reported as the intermediate host of E. leidyi (Prychitko and Nero 1983, Wolff 1984). More precisely, these intermediate host records for Nearctic $E$. leidyi will correspond to $M$. segerstralei and/or $M$. diluviana; $M$. relicta sensu stricto appears to be confined to north European fresh and brackish waters (Audzijonytė and Väinölä 2005). Definitive hosts include salmonid and coregonid fishes. Väinölä et al. (1994) postulated that the common usage of M. relicta group species as intermediate hosts defines $E$. leidyi and the $E$. bothniensis group as a clade of closely related species, because the known life cycles of all other Echinorhynchus spp. involve an amphipod intermediate host. Furthermore, these authors advanced an hypothesis of co-speciation of the acanthocephalans with their mysid hosts.

Both E. bothniensis and E. leidyi have a similar hooks formula and cement gland arrangement to a congener, E. truttae Schrank, 1788 found in salmoniform fishes of the Palaearctic. E. truttae utilises an amphipod (Gammarus pulex (L.)), rather than a mysid, as an intermediate host (Awachie 1966) and so is apparently biologically distinct from the $E$. bothniensis group and E. leidyi. Zdzitowiecki and Valtonen (1987) reported that $E$. bothniensis could be distinguished from E. truttae, because the latter has a longer proboscis and much shorter eggs. However, the diagnostic value of these characters was equivocal, since anatomical variability in $E$. truttae had never been assessed. The means to discriminate between the $E$. bothniensis group and $E$. truttae is of real significance to workers conducting faunistic surveys or other studies on these acanthocephalans. The two taxa share some of the same species of definitive host (e.g. Salmo trutta) (see Petrochenko 1956, Valtonen and Crompton 1990) and may well occur in sympatry, since their intermediate hosts have overlapping geographical ranges in northern Europe (see Pinkster 1978, Väinölä et al. 1994).

E. truttae is typically a parasite of salmoniform fishes and has been reported from a variety of species including S. trutta (e.g. Awachie 1966), S. alpinus (e.g. Dorucu et al. 1995), Salvelinus leucomaenis (Pallas) (Nagasawa et al. 1997), C. lavaretus (e.g. Petrochenko 1956), Thymallus thymallus (L.) (e.g. Petrochenko 1956), Thymallus arcticus baicalensis Dybowski (Baldanova and Pronin 1998, Baldanova 2000) and Oncorhynchus mykiss (Walbaum) (Dorucu et al. 1995, Holland and Kennedy 1997). E. truttae is found throughout Europe (including Ireland and the British Isles) and its range extends across Siberia all the way to the Bering Straits (Petrochenko 1956). Golvan (1994) suggested that E. truttae (sensu Zhukov 1960) from the Kurile Islands, northwest Pacific, may be another species.

The principal aims of the present study were: (i) to ascertain whether the two sibling species of the $E$. bothniensis group can be distinguished from each other, and from $E$. leidyi, using morphological characters; (ii) to review the taxonomy of $E$. bothniensis and $E$. 
'bothniensis'; (iii) to quantify morphological variability in $E$. truttae; and (iv) to identify the best characters for discriminating this taxon from the $E$. bothniensis sibling species and $E$. leidyi. Additionally, some observations on the ecology of $E$. truttae are reported.

\section{Material and methods}

\section{Material}

Table 1 provides a detailed list of all material studied, including accession numbers. A total of 19 specimens ( 7 males; 12 females) of Echinorhynchus truttae were collected from wild brown trout (Salmo trutta L.) from two streams (Loch Walton Burn and Loch Coulter Burn) in the River Carron catchment, central Scotland. The fish were caught by electro-fishing and were transported live to the laboratory where they were killed by a blow to the head and examined for acanthocephalan infection within 24 hours. Acanthocephalans found were washed and relaxed in refrigerated distilled water before being fixed in $75 \%$ alcohol. These acanthocephalans were identified as Echinorhynchus truttae using the keys in Petrochenko (1956). They were judged to be E. truttae, rather than members of the morphogically similar E. bothniensis group or E. leidyi, because the lotic environment they were collected from is unlikely to support populations of the lentic Mysis relicta, the intermediate host of the E. bothniensis group. Furthermore, the trout sampled were in their first year of life and so were unlikely to have spent any time outside their natal stream where they might potentially have been infected with $E$. bothniensis.

Table 1.

Material Studied.

\begin{tabular}{|c|c|c|c|c|c|c|}
\hline Species & Host & Locality & $\begin{array}{l}\text { Date } \\
\text { Collected }\end{array}$ & $\begin{array}{l}\text { Accession } \\
\text { Numbers }\end{array}$ & $\begin{array}{l}\text { ID Prefix in } \\
\text { Supplementary } \\
\text { Files }\end{array}$ & $\begin{array}{l}\text { Number of } \\
\text { Specimens }\end{array}$ \\
\hline E.truttae & Salmotrutta L. & $\begin{array}{l}\text { Drummore, } \\
\text { southwest } \\
\text { Scotland }\end{array}$ & NA & $\begin{array}{l}\mathrm{BM}(\mathrm{NH}) \\
1986.764-793\end{array}$ & $\mathrm{t} 1$. & $\begin{array}{l}74 \\
(45 \mathrm{f}, 29 \mathrm{~m})\end{array}$ \\
\hline E.truttae & S.trutta & $\begin{array}{l}\text { Loch Walton } \\
\text { Burn, River } \\
\text { Carron } \\
\text { catchment, } \\
\text { central } \\
\text { Scotland } \\
\text { (National Grid } \\
\text { Reference NS } \\
668 \text { 865) }\end{array}$ & $\begin{array}{l}\text { 24th June } \\
1996\end{array}$ & $\begin{array}{l}\mathrm{BM}(\mathrm{NH}) \\
2002.2 .4 .264- \\
275\end{array}$ & t2. & $\begin{array}{l}11 \\
(4 \mathrm{f}, 7 \mathrm{~m})\end{array}$ \\
\hline E.truttae & S.trutta & $\begin{array}{l}\text { Loch Coulter } \\
\text { Burn, River } \\
\text { Carron } \\
\text { catchment, } \\
\text { central } \\
\text { Scotland } \\
\text { (National Grid } \\
\text { Reference NS } \\
761 \text { 865) }\end{array}$ & $\begin{array}{l}\text { 20th } \\
\text { September } \\
1996\end{array}$ & $\begin{array}{l}\mathrm{BM}(\mathrm{NH}) \\
2002.2 .4 .276- \\
283\end{array}$ & t3. & $\begin{array}{l}8 \\
(8 \mathrm{f}, 0 \mathrm{~m})\end{array}$ \\
\hline
\end{tabular}




\begin{tabular}{|c|c|c|c|c|c|c|}
\hline E.bothniensis & $\begin{array}{l}\text { Osmeruseperlanus } \\
\text { L. }\end{array}$ & $\begin{array}{l}\text { Bothnian Bay, } \\
\text { Baltic Sea }\end{array}$ & $\begin{array}{l}\text { 13th July } \\
1985\end{array}$ & $\begin{array}{l}\text { BM (NH) } \\
1987.1070- \\
1074 \\
\text { (paratypes) }\end{array}$ & b1. & $\begin{array}{l}1 \\
(1 \mathrm{f}, 0 \mathrm{~m})\end{array}$ \\
\hline E.bothniensis & O.eperlanus & $\begin{array}{l}\text { Lake Keitele, } \\
\text { central Finland }\end{array}$ & $\begin{array}{l}10 \text { th } \\
\text { October } \\
1996\end{array}$ & $\begin{array}{l}\mathrm{BM}(\mathrm{NH}) \\
2002.2 \cdot 4.102- \\
122\end{array}$ & b2. & $\begin{array}{l}19 \\
(8 \mathrm{f}, 0 \mathrm{~m})\end{array}$ \\
\hline E.bothniensis & O.eperlanus & $\begin{array}{l}\text { Lake Keitele, } \\
\text { central Finland }\end{array}$ & $\begin{array}{l}26 \text { th } \\
\text { October } \\
1989\end{array}$ & $\begin{array}{l}\mathrm{BM}(\mathrm{NH}) \\
1989.1474- \\
1491\end{array}$ & b4. & $\begin{array}{l}13 \\
(6 \mathrm{f}, 7 \mathrm{~m})\end{array}$ \\
\hline $\begin{array}{l}\text { E.' } \\
\text { bothniensis' }\end{array}$ & $\begin{array}{l}\text { Salvelinusalpinus } \\
\text { (L.) }\end{array}$ & $\begin{array}{l}\text { Lake } \\
\text { Pulmankijärvi, } \\
\text { northern } \\
\text { Finland }\end{array}$ & $\begin{array}{l}\text { 14th June } \\
1989\end{array}$ & $\begin{array}{l}\mathrm{BM}(\mathrm{NH}) \\
1989.1241- \\
1248\end{array}$ & b5. & $\begin{array}{l}7 \\
(4 \mathrm{f}, 3 \mathrm{~m})\end{array}$ \\
\hline $\begin{array}{l}\text { E.' } \\
\text { bothniensis' }\end{array}$ & S.alpinus & $\begin{array}{l}\text { Lake } \\
\text { Pulmankijärvi, } \\
\text { northern } \\
\text { Finland }\end{array}$ & NA & $\begin{array}{l}\mathrm{BM}(\mathrm{NH}) \\
1989.1439- \\
1468\end{array}$ & b6. & $\begin{array}{l}2 \\
(2 \mathrm{f}, 0 \mathrm{~m})\end{array}$ \\
\hline $\begin{array}{l}\text { E.' } \\
\text { bothniensis' }\end{array}$ & $\begin{array}{l}\text { Coregonuslavaretus } \\
\text { (L.) }\end{array}$ & $\begin{array}{l}\text { Lake } \\
\text { Pulmankijärvi, } \\
\text { northern } \\
\text { Finland }\end{array}$ & NA & $\begin{array}{l}\mathrm{BM}(\mathrm{NH}) \\
1989.1259- \\
1270\end{array}$ & b7. & $\begin{array}{l}16 \\
(8 \mathrm{f}, 8 \mathrm{~m})\end{array}$ \\
\hline $\begin{array}{l}\text { E.' } \\
\text { bothniensis' }\end{array}$ & C.lavaretus & $\begin{array}{l}\text { Lake } \\
\text { Pulmankijärvi, } \\
\text { northern } \\
\text { Finland }\end{array}$ & $\begin{array}{l}\text { 14th-16th } \\
\text { June } 1989\end{array}$ & $\begin{array}{l}\mathrm{BM}(\mathrm{NH}) \\
1989.1406- \\
1420\end{array}$ & b8. & $\begin{array}{l}5 \\
(3 \mathrm{f}, 2 \mathrm{~m})\end{array}$ \\
\hline $\begin{array}{l}\text { E. ' } \\
\text { bothniensis' }\end{array}$ & $\begin{array}{l}\text { Platichthysflesus } \\
\text { (L.) }\end{array}$ & $\begin{array}{l}\text { Lake } \\
\text { Pulmankijärvi, } \\
\text { northern } \\
\text { Finland }\end{array}$ & $\begin{array}{l}\text { 11th June } \\
1990\end{array}$ & NA & b9. & $\begin{array}{l}4 \\
(3 \mathrm{f}, 1 \mathrm{~m})\end{array}$ \\
\hline E.leidyi & S.alpinus & $\begin{array}{l}\text { Kinguk Lake, } \\
\text { Northwest } \\
\text { Territories, } \\
\text { Canada } 64^{\circ} 40 \\
\text { 'N } 75^{\circ} 30^{\prime} \mathrm{W}\end{array}$ & $\begin{array}{l}27 \text { th } \\
\text { August } \\
1984\end{array}$ & $\begin{array}{l}\text { CMNPA 1985- } \\
0146\end{array}$ & 11. & $\begin{array}{l}3 \\
(3 \mathrm{f}, 0 \mathrm{~m})\end{array}$ \\
\hline E.leidyi & C.lavaretus & $\begin{array}{l}\text { Southern } \\
\text { Indian Lake, } \\
\text { Manitoba, } \\
\text { Canada } 58^{\circ} 45 \\
\text { 'N } 98^{\circ} 55^{\prime} \mathrm{W}\end{array}$ & $\begin{array}{l}\text { 8th June } \\
1982\end{array}$ & $\begin{array}{l}\text { CMNPA 1985- } \\
0138\end{array}$ & 12. & $\begin{array}{l}5 \\
(0 \mathrm{f}, 5 \mathrm{~m})\end{array}$ \\
\hline E.leidyi & S.alpinus & $\begin{array}{l}\text { Unnamed lake, } \\
\text { Northwest } \\
\text { Territories, } \\
\text { Canada } 64^{\circ} 26 \\
\text { 'N } 77^{\circ} 45^{\prime} \mathrm{W}\end{array}$ & $\begin{array}{l}29 \text { th } \\
\text { August } \\
1984\end{array}$ & $\begin{array}{l}\text { CMNPA 1985- } \\
0149\end{array}$ & 13. & $\begin{array}{l}5 \\
(0 \mathrm{f}, 5 \mathrm{~m})\end{array}$ \\
\hline
\end{tabular}

A series of $E$. truttae (74 specimens; 45 females; 29 males) collected by $\mathrm{Dr}$ A Pike, University of Aberdeen, from S. trutta from Drummore, on the south-west coast of Scotland, held in the spirit collection of the Natural History Museum was also studied. Most of these acanthocephalans had well everted probosces and displayed no tegumental folding, suggesting that they had been relaxed in water before being fixed.

All of the specimens of the E. bothniensis group studied were collected between 1985 and 1997 by Professor E. T. Valtonen of the University of Jyväskylä and deposited in the spirit collection of The Natural History Museum. Some of this material had been fully relaxed in water prior to fixation. Most of the $E$. bothniensis material came from one host species, $O$. eperlanus, from the freshwater Lake Keitele, central Finland. This population of $E$. bothniensis is thought to have been isolated from conspecifics in the Bothnian Bay for at 
least 6,000 years (Väinölä et al. 1994). Five paratypes of $E$. bothniensis (BM(NH) 1987.1070-1074) from O. eperlanus from the Bothnian Bay were also examined, but only one female worm was in a suitable condition for measuring hook morphometrics.

E. 'bothniensis' is known only from Lake Pulmankijärvi in northern Lapland, on the FinnishNorwegian border. This freshwater lake lies 17 metres above sea level and drains into the Barents Sea. Samples of E. 'bothniensis' were obtained from the following hosts: Salvelinus alpinus (L.), Coregonus lavaretus (L.) and Platichthys flesus (L.).

In addition to the northern European material described above, voucher specimens of the Nearctic E. leidyi from the Canadian Museum of Nature were also examined. These acanthocephalans were collected by Shostak et al. (1986) during their extensive survey of morphological variability in E. gadi, E. leidyi and E. salmonis Müller, 1784 from northern Canada.

\section{Light microscopy}

The specimens of $E$. leidyi from the Canadian Museum of Nature had been fixed in formalin-acetic acid-alcohol (FAA), stained with Semichon's carmine and permanently mounted in Permount (Fisher Scientific). All other acanthocephalans were prepared for light microscopy by dehydration through an alcohol series followed by clearing in lactophenol. Measurements were made with aid of a digitizing tablet (KS 100, Version 3, Carl Zeiss Vision). Hook morphometric data were recorded from one longitudinal row in which all of the hooks were visible in profile using the method described by Wayland (2010). Morphometric and meristic data were collected during a PhD studentship (Wayland 2002).

\section{Morphological data analysis}

Statistical analysis and visualization of morphometric and meristic data were performed using the $R$ language and environment ( $R$ Core Team 2012). Boxplots augmented with strip charts were created using the $R$ package beeswarm (Eklund 2012). Proboscis profiler (Wayland 2010) was used to analyse both intra and interspecific variation in hook measurements. Proboscis profiler, based on the meristogram of Huffman and Bullock (1975), was developed to detect morphological heterogeneity in collections of superficially similar acanthocephalan worms based on the multivariate statistical analysis of proboscis hook dimensions. For a detailed description of this tool with examples, please refer to Wayland (2010). In brief, the Proboscis profiler algorithm is composed of the following sequential steps:

1. Proboscis profiler requires blade length and base width measurements from each of the hooks in at least one longitudinal row of hooks per specimen. In each longitudinal row hooks are numbered sequentially, starting with the most distal hook.

2. Hook position is standardized. Counted position number of each hook in a given row is multiplied by 100 and divided by $n+1$, where $n=$ the total number of hooks 
in the row and the constant 1 is a corrective factor for centring the data-points in graphs.

3. A moving average (arithmetic mean) routine is applied to the data from each row of hooks and considers a user-defined segment of the percent-position axis for each measurement (length and base). The segment advances through the data from anterior to posterior in $1 \%$ increments.

4. Unsupervised pattern recognition using principal component analysis.

5. Hierarchical clustering of the first two principal components from step 4.

\section{Ecological data analysis}

For each of the two host populations studied (Loch Walton Burn and Loch Coulter Burn), Quantitative Parasitology (Rózsa et al. 2000, Reiczigel 2003) was used to calculate an exact confidence interval for the prevalence of infection (using the Sterne method), a bootstrap confidence interval for mean abundance and the aggregation index (variance/ mean). The R package fitdistrplus (Delignette-Muller et al. 2013) was used to determine whether a Poisson or a negative binomial distribution provided the best description of the occurrence of Echinorhynchus truttae in its definitive host populations.

\section{Data resources}

All data collected for this study are available as supplementary files.

\section{Morphological data}

Standard morphometric and meristic data for female and male acanthocephalans can be found in Suppl. materials 1, 2 respectively. Egg and acanthor dimensions are listed in Suppl. material 3. Hook measurement data for female and male acanthocephalans (Suppl. materials 4,5 respectively) are in a file format suitable as input to the Proboscis Profiler software (Wayland 2010).

\section{Ecological data}

Suppl. materials 6, 7 contain data on the occurrence of $E$. truttae in samples of its definitive host S. trutta from Loch Coulter and Loch Walton respectively. For each fish examined, fork length and intensity of infection were recorded.

\section{Results}

\section{Variation in conventional morphological characters}

Initially an assessment was made of intraspecific and interspecific variation in conventional morphological characters, i.e. those characters used by most acanthocephalan taxonomists in the differential diagnosis of Echinorhynchus species. Summaries of these 
variables for the female and male acanthocephalans examined in this study are provided in Tables 2, 3 respectively. Data for the three E. truttae populations (Loch Walton Burn, Loch Coulter Burn and Drummore) have been pooled, because, in the absence of any inter-site morphological variability, these acanthocephalans were assumed to be conspecific. Additionally, for comparative purposes, Tables 2, 3 contain data for E. bothniensis from $O$. eperlanus in the Bothnian Bay (original description by Zdzitowiecki and Valtonen 1987) and an extensive collection of $E$. leidyi from various fishes across northern Canadian waters (Shostak et al. 1986). It is important to note that these additional data were recorded from acanthocephalans prepared for light microscopy using methods different from those employed in the current study, although in all studies acanthocephalans were relaxed in fresh water prior to fixation to evert proboscides. Zdzitowiecki and Valtonen (1987) fixed their samples of $E$. bothniensis in alcohol and examined them as wet mounts, similarly to the current study, however they used creosote rather than lactophenol as a clearing agent. By contrast, Shostak et al. (1986) fixed their samples in formalin-acetic acid-alcohol (FAA), stained them with acetocarmine and mounted them in synthetic resin.

Table 2.

Morphometrics of female Echinorhynchus bothniensis, E. 'bothniensis', E. leidyi and E. truttae (range; mean + standard deviation and sample size in parentheses). Data available in Suppl. materials 1,3 .

\begin{tabular}{|c|c|c|c|c|c|}
\hline & $\begin{array}{l}\text { E.bothniensis } \\
\text { Bothnian Bay } \\
\text { (Zdzitowiecki and } \\
\text { Valtonen, 1987) }\end{array}$ & $\begin{array}{l}\text { E.bothniensis } \\
\text { Lake Keitele (this } \\
\text { study) }\end{array}$ & $\begin{array}{l}\text { E. 'bothniensis' } \\
\text { Lake Pulmankijärvi } \\
\text { (this study) }\end{array}$ & $\begin{array}{l}\text { E.leidyi } \\
\text { Northern } \\
\text { Canada } \\
\text { (Shostak et } \\
\text { al., 1986) }\end{array}$ & $\begin{array}{l}\text { E.truttae } \\
\text { Scotland } \\
\text { (this study) }\end{array}$ \\
\hline $\begin{array}{l}\text { Body length } \\
(\mathrm{mm})\end{array}$ & $\begin{array}{l}10.5-27.1 \\
(-; 38)\end{array}$ & $\begin{array}{l}10.1-25.1 \\
(16.0 \pm 4.44 ; 14)\end{array}$ & $\begin{array}{l}8.2-15.8 \\
(10.9 \pm 2.28 ; 18)\end{array}$ & $\begin{array}{l}3.9-31.6 \\
(16.4 \pm 4.36 \\
476)\end{array}$ & $\begin{array}{l}9.0-18.9 \\
(14.0 \pm 2.00 \\
56)\end{array}$ \\
\hline $\begin{array}{l}\text { Body width } \\
(\mathrm{mm})\end{array}$ & $\begin{array}{l}1.12-3.13 \\
(-; 38)\end{array}$ & $\begin{array}{l}1.14-2.76 \\
(1.89 \pm 0.50 ; 14)\end{array}$ & $\begin{array}{l}0.71-2.72 \\
(1.32 \pm 0.50 ; 20)\end{array}$ & $\begin{array}{l}0.60-3.0 \\
(1.2 \pm 0.26 \\
478)\end{array}$ & $\begin{array}{l}0.85-2.02 \\
(1.19 \pm 0.25 \\
56)\end{array}$ \\
\hline $\begin{array}{l}\text { Body length/ } \\
\text { width }\end{array}$ & - & $\begin{array}{l}5.6-11.8 \\
(8.6 \pm 1.52 ; 14)\end{array}$ & $\begin{array}{l}3.8-13.8 \\
(9.2 \pm 2.34 ; 18)\end{array}$ & $\begin{array}{l}4.3-27.4 \\
(13.7 \pm 3.40 \\
466)\end{array}$ & $\begin{array}{l}7.4-16.5 \\
(12.1 \pm 2.02 \\
56)\end{array}$ \\
\hline $\begin{array}{l}\text { Proboscis } \\
\text { length }\end{array}$ & $\begin{array}{l}660-940 \\
(846 \pm 60 ; 38)\end{array}$ & $\begin{array}{l}611-787 \\
(717 \pm 56.6 ; 7)\end{array}$ & $\begin{array}{l}711-904 \\
(823 \pm 77.3 ; 5)\end{array}$ & $\begin{array}{l}733-1335 \\
(1037 \pm 116.6 \\
508)\end{array}$ & $\begin{array}{l}869-1188 \\
(1009 \pm 59.7 \\
56)\end{array}$ \\
\hline $\begin{array}{l}\text { Proboscis } \\
\text { width }\end{array}$ & $\begin{array}{l}230-290 \\
(264 \pm 15 ; 38)\end{array}$ & $\begin{array}{l}248-344 \\
(308 \pm 33.2 ; 11)\end{array}$ & $\begin{array}{l}213-334 \\
(285 \pm 34.3 ; 19)\end{array}$ & $\begin{array}{l}187-355 \\
(274 \pm 31.0 \\
508)\end{array}$ & $\begin{array}{l}249-359 \\
(309 \pm 22.2 \\
56)\end{array}$ \\
\hline $\begin{array}{l}\text { Proboscis } \\
\text { length/width }\end{array}$ & $\begin{array}{l}2.82-3.67 \\
(3.21 \pm 0.21 ; 38)\end{array}$ & $\begin{array}{l}2.03 \pm 2.95 \\
(2.47 \pm 0.370 ; 7)\end{array}$ & $\begin{array}{l}2.61-3.77 \\
(3.04 \pm 0.500 ; 5)\end{array}$ & $\begin{array}{l}2.64-5.98 \\
(3.81 \pm 0.414 \\
508)\end{array}$ & $\begin{array}{l}2.73-3.93 \\
(3.28 \pm \\
0.289 ; 56)\end{array}$ \\
\hline $\begin{array}{l}\text { Number of } \\
\text { rows of hooks }\end{array}$ & $18-22$ & $\begin{array}{l}18-21 \\
(19.2 \pm 0.98 ; 14)\end{array}$ & $\begin{array}{l}18-22 \\
(19.5 \pm 1.07 ; 19)\end{array}$ & $\begin{array}{l}14-23 \\
(18.1 \pm 1.66 \\
508)\end{array}$ & $\begin{array}{l}16-22 \\
(19.6 \pm 1.44 \\
57)\end{array}$ \\
\hline
\end{tabular}




\begin{tabular}{|c|c|c|c|c|c|}
\hline $\begin{array}{l}\text { Number of } \\
\text { hooks per row }\end{array}$ & $11-15$ & $\begin{array}{l}11-12 \\
(11.9 \pm 0.35 ; 8)\end{array}$ & $\begin{array}{l}12-15 \\
(13.2 \pm 1.10 ; 5)\end{array}$ & $\begin{array}{l}10-17 \\
(14.1 \pm 1.11 \\
508)\end{array}$ & $\begin{array}{l}12-17 \\
(14.6 \pm 0.98 \\
57)\end{array}$ \\
\hline $\begin{array}{l}\text { Maximum } \\
\text { length of hook } \\
\text { blade }\end{array}$ & $\begin{array}{l}57-72 \\
(64 \pm 3.0 ; 38)\end{array}$ & $\begin{array}{l}57-66 \\
(61 \pm 3.6 ; 4)\end{array}$ & $\begin{array}{l}64-68 \\
(65 \pm 2.1 ; 3)\end{array}$ & $\begin{array}{l}52-84 \\
(70 \pm 4.8 ; 508)\end{array}$ & $\begin{array}{l}68-91 \\
(78 \pm 3.8 \\
46)\end{array}$ \\
\hline $\begin{array}{l}\text { Proboscis } \\
\text { receptacle } \\
\text { length }\end{array}$ & $\begin{array}{l}1080-1850 \\
(1497 \pm 176 ; 38)\end{array}$ & $\begin{array}{l}1237-2195 \\
(1615 \pm 249 ; 14)\end{array}$ & $\begin{array}{l}668-1922 \\
(1284 \pm 323 ; 20)\end{array}$ & - & $\begin{array}{l}1486-2855 \\
(1901 \pm 287 \\
56)\end{array}$ \\
\hline $\begin{array}{l}\text { Proboscis } \\
\text { receptacle } \\
\text { width }\end{array}$ & $\begin{array}{l}300-430 \\
(366 \pm 33 ; 38)\end{array}$ & $\begin{array}{l}336-618 \\
(436 \pm 77 ; 14)\end{array}$ & $\begin{array}{l}167-431 \\
(296 \pm 63 ; 20)\end{array}$ & - & $\begin{array}{l}318 \pm 616 \\
(407 \pm 77 \\
56)\end{array}$ \\
\hline $\begin{array}{l}\text { Lemniscus } \\
\text { length }\end{array}$ & $\begin{array}{l}870-1890 \\
(-; 38)\end{array}$ & $\begin{array}{l}958-1963 \\
(1462 \pm 323 ; 14)\end{array}$ & $\begin{array}{l}510-1543 \\
(901 \pm 290 ; 19)\end{array}$ & - & $\begin{array}{l}935-2434 \\
(1670 \pm 293 \\
56)\end{array}$ \\
\hline $\begin{array}{l}\text { Lemniscus } \\
\text { width }\end{array}$ & $\begin{array}{l}220-540 \\
(-; 38)\end{array}$ & $\begin{array}{l}212-616 \\
(361 \pm 111 ; 14)\end{array}$ & $\begin{array}{l}99-441 \\
(266 \pm 90 ; 19)\end{array}$ & - & $\begin{array}{l}201-693 \\
(350 \pm 93 \\
56)\end{array}$ \\
\hline $\begin{array}{l}\text { Genital } \\
\text { complex } \\
\text { length }\end{array}$ & $\begin{array}{l}1480-2270 \\
(1846 \pm 201 ; 38)\end{array}$ & $\begin{array}{l}1575-2104 \\
(1912 \pm 186 ; 6)\end{array}$ & $\begin{array}{l}991-1669 \\
(1356 \pm 193 ; 12)\end{array}$ & - & $\begin{array}{l}1357-2761 \\
(1792 \pm 289 \\
25)\end{array}$ \\
\hline $\begin{array}{l}\text { Uterine bell } \\
\text { length }\end{array}$ & - & $\begin{array}{l}375-734 \\
(551 \pm 147 ; 6)\end{array}$ & $\begin{array}{l}265-555 \\
(368 \pm 93 ; 12)\end{array}$ & - & $\begin{array}{l}429-878 \\
(568 \pm 93 \\
25)\end{array}$ \\
\hline Uterus length & - & $\begin{array}{l}1060-1749 \\
(1314 \pm 212 ; 8)\end{array}$ & $\begin{array}{l}646-1203 \\
(902 \pm 158 ; 13)\end{array}$ & - & $\begin{array}{l}614-1592 \\
(1003 \pm 191 \\
42)\end{array}$ \\
\hline Uterus width & - & $\begin{array}{l}110-237 \\
(161 \pm 44.1 ; 11)\end{array}$ & $\begin{array}{l}41-157 \\
(71 \pm 34.1 ; 16)\end{array}$ & - & $\begin{array}{l}56-219 \\
(110 \pm 30.1 \\
55)\end{array}$ \\
\hline Vagina length & - & $\begin{array}{l}218-344 \\
(273 \pm 42.9 ; 14)\end{array}$ & $\begin{array}{l}183-281 \\
(221 \pm 25.6 ; 14)\end{array}$ & - & $\begin{array}{l}234-394 \\
(294 \pm 29.7 \\
56)\end{array}$ \\
\hline Vagina width & - & $\begin{array}{l}62-144 \\
(103 \pm 26.1 ; 14)\end{array}$ & $\begin{array}{l}65-98 \\
(80 \pm 10.3 ; 14)\end{array}$ & - & $\begin{array}{l}72-149 \\
(109 \pm 15.2 \\
56)\end{array}$ \\
\hline $\begin{array}{l}\text { Vaginal } \\
\text { sphincter } \\
\text { width }\end{array}$ & - & $\begin{array}{l}97-208 \\
(142 \pm 33.9 ; 14)\end{array}$ & $\begin{array}{l}61-125 \\
(82 \pm 19.3 ; 15)\end{array}$ & - & $\begin{array}{l}91-182 \\
(126 \pm 19.4 \\
56)\end{array}$ \\
\hline $\begin{array}{l}\text { Spincter width } \\
\text { to vagina } \\
\text { width ratio }\end{array}$ & - & $\begin{array}{l}1.04-1.97 \\
(1.41 \pm 0.271 ; 14)\end{array}$ & $\begin{array}{l}0.73-1.28 \\
(1.02 \pm 0.184 ; 14)\end{array}$ & - & $\begin{array}{l}0.88-2.01 \\
(1.17 \pm \\
0.161 ; 56)\end{array}$ \\
\hline Egg length & $\begin{array}{l}140-168 \\
(156 \pm 7 ; 38)\end{array}$ & $\begin{array}{l}127-166 \\
(148 \pm 12.6 ; 15)\end{array}$ & $\begin{array}{l}121-152 \\
(137 \pm 11.4 ; 9)\end{array}$ & $\begin{array}{l}90-135 \\
(115 \pm 8.2 \\
134)\end{array}$ & $\begin{array}{l}120-173 \\
(140 \pm 11.0 \\
117)\end{array}$ \\
\hline Egg width & $\begin{array}{l}22-29 \\
(25 \pm 1 ; 38)\end{array}$ & $\begin{array}{l}19-31(23 \pm 3.1 \\
15)\end{array}$ & $\begin{array}{l}19-23 \\
(21 \pm 1.2 ; 9)\end{array}$ & - & $\begin{array}{l}22-34 \\
(27 \pm 2.2 \\
117)\end{array}$ \\
\hline
\end{tabular}




\begin{tabular}{|c|c|c|c|c|c|}
\hline $\begin{array}{l}\text { Acanthor } \\
\text { length }\end{array}$ & - & $\begin{array}{l}67-80 \\
(73 \pm 3.5 ; 15)\end{array}$ & $\begin{array}{l}67-78 \\
(74 \pm 3.9 ; 9)\end{array}$ & - & $\begin{array}{l}70-90 \\
(80 \pm 4.4 \\
117)\end{array}$ \\
\hline Acanthor width & - & $\begin{array}{l}14-19 \\
(17 \pm 1.5 ; 15)\end{array}$ & $\begin{array}{l}14-19 \\
(17 \pm 1.5 ; 9)\end{array}$ & - & $\begin{array}{l}17-24 \\
(20 \pm 1.4 \\
117)\end{array}$ \\
\hline
\end{tabular}

Table 3.

Morphometrics of male Echinorhynchus bothniensis, E. 'bothniensis', E. leidyi and E. truttae (range; mean + standard deviation and sample size in parentheses). Data available in Suppl. material 2.

\begin{tabular}{|c|c|c|c|c|c|}
\hline & $\begin{array}{l}\text { E.bothniensis } \\
\text { Bothnian Bay } \\
\text { (Zdzitowiecki and } \\
\text { Valtonen, 1987) }\end{array}$ & $\begin{array}{l}\text { E.bothniensis } \\
\text { Lake Keitele } \\
\text { (this study) }\end{array}$ & $\begin{array}{l}\text { E. 'bothniensis' } \\
\text { Lake } \\
\text { Pulmankijärvi } \\
\text { (this study) }\end{array}$ & $\begin{array}{l}\text { E.leidyi } \\
\text { Northern } \\
\text { Canada } \\
\text { (Shostak et } \\
\text { al., 1986) }\end{array}$ & $\begin{array}{l}\text { E.truttae } \\
\text { Scotland } \\
\text { (this study) }\end{array}$ \\
\hline Body length (mm) & $8.9-15.8$ & $\begin{array}{l}7.4-15.9 \\
(10.9 \pm 2.9 ; 16)\end{array}$ & $\begin{array}{l}4.5-9.7 \\
(7.3 \pm 1.6 ; 14)\end{array}$ & $\begin{array}{l}5.1-19.7 \\
(10.3 \pm 2.51 \\
360)\end{array}$ & $\begin{array}{l}7.2-10.9 \\
(8.9 \pm 1.09 \\
32)\end{array}$ \\
\hline Body width (mm) & $1.13-2.39$ & $\begin{array}{l}0.93-2.17 \\
(1.47 \pm 0.36 ; 14)\end{array}$ & $\begin{array}{l}0.58-1.78 \\
(1.04 \pm 0.37 ; 14)\end{array}$ & $\begin{array}{l}0.6-1.9 \\
(1.0 \pm 0.20 \\
353)\end{array}$ & $\begin{array}{l}0.69-1.32 \\
(0.90 \pm 0.12 \\
32\end{array}$ \\
\hline Body length/width & - & $\begin{array}{l}5.5-10.3 \\
(7.8 \pm 1.42 ; 14)\end{array}$ & $\begin{array}{l}4.9-10.2 \\
(7.4 \pm 1.40 ; 14)\end{array}$ & $\begin{array}{l}5.6-21.0 \\
(10.7 \pm 3.03 \\
352)\end{array}$ & $\begin{array}{l}6.7-12.2 \\
(10.0 \pm 1.29 \\
32)\end{array}$ \\
\hline $\begin{array}{l}\text { Reproductive } \\
\text { system length }(\mathrm{mm})\end{array}$ & - & $\begin{array}{l}5.1-11.0 \\
(7.4 \pm 2.17 ; 13)\end{array}$ & $\begin{array}{l}3.0-6.3 \\
(4.8 \pm 1.08 ; 14)\end{array}$ & - & $\begin{array}{l}4.0-6.6 \\
(5.4 \pm 0.69 \\
32)\end{array}$ \\
\hline Proboscis length & $\begin{array}{l}690-830 \\
(756 \pm 36 ; 50)\end{array}$ & $\begin{array}{l}617-751 \\
(683 \pm 42.8 ; 13)\end{array}$ & - & $\begin{array}{l}658-1203 \\
(930 \pm 93.3 \\
381)\end{array}$ & $\begin{array}{l}733-1019 \\
(903 \pm 59.6 \\
32)\end{array}$ \\
\hline Proboscis width & $\begin{array}{l}220-280 \\
(240 \pm 13 ; 50)\end{array}$ & $\begin{array}{l}204-329 \\
(265 \pm 37.8 ; 16)\end{array}$ & $\begin{array}{l}204-287 \\
(256 \pm 24.6 ; 8)\end{array}$ & $\begin{array}{l}176-314 \\
(245 \pm 27.6 \\
381)\end{array}$ & $\begin{array}{l}205-326 \\
(264 \pm 29.0 \\
32)\end{array}$ \\
\hline $\begin{array}{l}\text { Proboscis length/ } \\
\text { width }\end{array}$ & $\begin{array}{l}2.69-3.51 \\
(3.16 \pm 0.22 ; 50)\end{array}$ & $\begin{array}{l}2.00-3.16 \\
(2.51 \pm 0.327 ; 13)\end{array}$ & - & $\begin{array}{l}2.57-5.24 \\
(3.83 \pm 0.424 \\
381)\end{array}$ & $\begin{array}{l}2.67-4.07 \\
(3.46 \pm \\
0.381 ; 32)\end{array}$ \\
\hline $\begin{array}{l}\text { Number of rows of } \\
\text { hooks }\end{array}$ & $17-20$ & $\begin{array}{l}17-21 \\
(19.0 \pm 1.50 ; 17)\end{array}$ & $\begin{array}{l}18-22 \\
(19.4 \pm 1.2610)\end{array}$ & $\begin{array}{l}12-22 \\
(17.5 \pm 1.77 \\
381)\end{array}$ & $\begin{array}{l}16-22 \\
(18.7 \pm 1.45 \\
35)\end{array}$ \\
\hline $\begin{array}{l}\text { Number of hooks } \\
\text { per row }\end{array}$ & $11-14$ & $\begin{array}{l}11-13 \\
(11.9 \pm 0.59 ; 15)\end{array}$ & - & $\begin{array}{l}10-16 \\
(13.4 \pm 0.98 \\
381)\end{array}$ & $\begin{array}{l}11-15 \\
(14.0 \pm 0.95 \\
35)\end{array}$ \\
\hline $\begin{array}{l}\text { Maximum length of } \\
\text { hook blade }\end{array}$ & $\begin{array}{l}55-71 \\
(62 \pm 4 ; 50)\end{array}$ & $\begin{array}{l}50-61 \\
(57 \pm 3.9 ; 6)\end{array}$ & - & $\begin{array}{l}45-82 \\
(64 \pm 4.8 \\
381)\end{array}$ & $\begin{array}{l}67-84 \\
(75 \pm 3.7 \\
26)\end{array}$ \\
\hline
\end{tabular}




\begin{tabular}{|c|c|c|c|c|c|}
\hline $\begin{array}{l}\text { Proboscis } \\
\text { receptacle length }\end{array}$ & $\begin{array}{l}1140-1800 \\
(1452 \pm 137 ; 50)\end{array}$ & $\begin{array}{l}1042-1982 \\
(1559 \pm 231 ; 17)\end{array}$ & $\begin{array}{l}913-1262 \\
(1086 \pm 125 ; 13)\end{array}$ & - & $\begin{array}{l}1376-2384 \\
(1779 \pm 199 \\
32)\end{array}$ \\
\hline $\begin{array}{l}\text { Proboscis } \\
\text { receptacle width }\end{array}$ & $\begin{array}{l}240-350 \\
(303 \pm 27 ; 50)\end{array}$ & $\begin{array}{l}141-402 \\
(332 \pm 67 ; 17)\end{array}$ & $\begin{array}{l}154-345 \\
(257 \pm 62.6 ; 14)\end{array}$ & - & $\begin{array}{l}278-499 \\
(369 \pm 41.9 \\
32)\end{array}$ \\
\hline Lemniscus length & $720-1470$ & $\begin{array}{l}756-1678 \\
(1219 \pm 281 ; 15)\end{array}$ & $\begin{array}{l}496-977 \\
(717 \pm 157 ; 11)\end{array}$ & - & $\begin{array}{l}1172-1775 \\
(1468 \pm 164 \\
32)\end{array}$ \\
\hline Lemniscus width & $150-480$ & $\begin{array}{l}173-553 \\
(326 \pm 106 ; 15)\end{array}$ & $\begin{array}{l}107-268 \\
(207 \pm 54.3 ; 12)\end{array}$ & - & $\begin{array}{l}135-390 \\
(288 \pm 58.3 \\
32)\end{array}$ \\
\hline $\begin{array}{l}\text { Anterior testes } \\
\text { length }\end{array}$ & $800-1680$ & $\begin{array}{l}761-1682 \\
(1172 \pm 332 ; 12)\end{array}$ & $\begin{array}{l}403-934 \\
(649 \pm 165 ; 13)\end{array}$ & - & $\begin{array}{l}707-1249 \\
(1050 \pm 126 \\
28)\end{array}$ \\
\hline $\begin{array}{l}\text { Anterior testes } \\
\text { width }\end{array}$ & $370-670$ & $\begin{array}{l}289-831 \\
(476 \pm 145 ; 12)\end{array}$ & $\begin{array}{l}136-447 \\
(312 \pm 88.0 ; 13)\end{array}$ & - & $\begin{array}{l}394-637 \\
(513 \pm 70.0 \\
28)\end{array}$ \\
\hline $\begin{array}{l}\text { Posterior testes } \\
\text { length }\end{array}$ & $810-1700$ & $\begin{array}{l}686-1602 \\
(1069 \pm 295 ; 12)\end{array}$ & $\begin{array}{l}387-929 \\
(640 \pm 161 ; 13)\end{array}$ & - & $\begin{array}{l}694-1198 \\
(975 \pm 136 \\
28)\end{array}$ \\
\hline $\begin{array}{l}\text { Posterior testes } \\
\text { width }\end{array}$ & $300-680$ & $\begin{array}{l}306-837 \\
(475 \pm 158 ; 12)\end{array}$ & $\begin{array}{l}197-471 \\
(334 \pm 84 ; 13)\end{array}$ & - & $\begin{array}{l}394-591 \\
(506 \pm 55 \\
28)\end{array}$ \\
\hline Cement gland width & - & $\begin{array}{l}178-954 \\
(356 \pm 207 ; 17)\end{array}$ & $\begin{array}{l}164-404 \\
(282 \pm 84 ; 14)\end{array}$ & - & $\begin{array}{l}198-575 \\
(365 \pm 83 \\
32)\end{array}$ \\
\hline $\begin{array}{l}\text { Saefftigen's pouch } \\
\text { length }\end{array}$ & $750-1050$ & $\begin{array}{l}659-1413 \\
(925 \pm 227 ; 17)\end{array}$ & $\begin{array}{l}500-871 \\
(684 \pm 117 ; 13)\end{array}$ & - & $\begin{array}{l}538-854 \\
(733 \pm 77 \\
32)\end{array}$ \\
\hline $\begin{array}{l}\text { Saefftigen's pouch } \\
\text { width }\end{array}$ & $160-270$ & $\begin{array}{l}116-336 \\
(227 \pm 72 ; 17)\end{array}$ & $\begin{array}{l}101-237 \\
(165 \pm 45 ; 13)\end{array}$ & - & $\begin{array}{l}187-374 \\
(288 \pm 44 \\
32)\end{array}$ \\
\hline Penis width & $\begin{array}{l}85-113 \\
(98 \pm 7 ; 50)\end{array}$ & $\begin{array}{l}50-105 \\
(79 \pm 16 ; 16)\end{array}$ & $\begin{array}{l}45-89 \\
(63 \pm 12 ; 9)\end{array}$ & - & $\begin{array}{l}66-110 \\
(85 \pm 11 ; 32)\end{array}$ \\
\hline $\begin{array}{l}\text { Bursal sucker } \\
\text { diameter }\end{array}$ & - & $\begin{array}{l}137-219 \\
(182 \pm 23 ; 11)\end{array}$ & $\begin{array}{l}135-191 \\
(164 \pm 16 ; 10)\end{array}$ & - & $\begin{array}{l}123-197 \\
(152 \pm 20 \\
15)\end{array}$ \\
\hline
\end{tabular}

The extent of intraspecific morphological variability for the taxa studied can be seen in Tables 2, 3. The mean and range of values for each morphometric are very similar for both E. bothniensis population, i.e. the Bothnian Bay and Lake Keitele. An analysis of the cause of intraspecific variation in morphological characters was attempted for $E$. truttae only, as sample numbers for the other taxa were considered to be too small for a meaningful statistical analysis. All anatomical characters common to both sexes are larger in females than males (compare data in tables Tables 2, 3 and also see boxplots in Suppl. material 8). Sexual dimporphism is also clearly apparent in a principal components analysis of conventional morphological characters (Fig. 1a). There is considerable separation of 
females from males in the first principal component, which accounts for $36 \%$ of the variation in the dataset. The variables contributing most to the separation of the two sexes (i.e. those with the highest loadings for principal component one) are: lemniscus length, proboscis receptacle length and width, body length and proboscis length and width (Fig. 1b] Body size is positively correlated (Bonferroni corrected p-value $<0.05$ ) with the size of several anatomical characters of female E. truttae (Table 4), namely, body width $\left(r^{2}=0.257\right.$ ), proboscis length $\left(r^{2}=0.317\right)$, proboscis receptacle length $\left(r^{2}=0.284\right)$, lemniscus length $\left(r^{2}\right.$ $=0.364)$, lemniscus width $\left(r^{2}=0.237\right)$, vagina width $\left(r^{2}=0.246\right)$ and vaginal sphincter width $(r$ ${ }^{2}=0.251$ ). In male E. truttae (Table 5 ), a significant positive correlation with body length is only demonstrated for the length of the reproductive system $\left(r^{2}=0.876\right)$, lemniscus length $\left(r^{2}=0.487\right)$ and the length of the testes $\left(r^{2}=0.346\right.$ for anterior testis; $r^{2}=0.469$ for posterior testis). Evidence of morphological variation in $E$. truttae between the three sample sites was not found, even after taking sexual dimorphism into account.

Table 4.

Correlation of morphometric variables with body length in female Echinorhynchus truttae. Correlation measured by Pearson's product-moment correlation coefficient ( $r$ ). The raw $p$ value is the probability that the sample correlation coefficient could have come from a population with a correlation coefficient of zero. The Bonferroni correction was used to control the family wise error rate across multiple tests of significance. Data available in Suppl. material 1.

\begin{tabular}{|l|l|l|l|l|}
\hline Variable & $\mathbf{n}$ & $\mathbf{r}$ & raw p & Bonferroni p \\
\hline Body width & 56 & 0.507 & 0.000066 & $\mathbf{0 . 0 0 0 9 9 7}$ \\
\hline Proboscis length & 56 & 0.563 & 0.000006 & $\mathbf{0 . 0 0 0 0 9 2}$ \\
\hline Proboscis width & 56 & 0.041 & 0.763773 & 1.000000 \\
\hline Proboscis receptacle length & 56 & 0.533 & 0.000023 & $\mathbf{0 . 0 0 0 3 4 6}$ \\
\hline Proboscis receptacle width & 56 & 0.375 & 0.004442 & 0.066630 \\
\hline Lemniscus length & 56 & 0.603 & $<0.000001$ & $\mathbf{0 . 0 0 0 0 1 3}$ \\
\hline Lemniscus width & 56 & 0.487 & 0.000142 & $\mathbf{0 . 0 0 2 1 2 8}$ \\
\hline Genital complex length & 25 & 0.438 & 0.028697 & 0.430462 \\
\hline Uterine bell length & 25 & 0.266 & 0.198106 & 1.000000 \\
\hline Uterus length & 42 & 0.376 & 0.014200 & 0.212997 \\
\hline Uterus width & 55 & 0.123 & 0.369147 & 1.000000 \\
\hline Vagina length & 56 & 0.273 & 0.041850 & 0.627757 \\
\hline Vagina width & 56 & 0.496 & 0.000100 & $\mathbf{0 . 0 0 1 5 0 0}$ \\
\hline Vaginal sphincter width & 56 & 0.501 & 0.000085 & $\mathbf{0 . 0 0 1 2 8 1}$ \\
\hline Maximum length of hook blade & 46 & 0.267 & 0.072923 & 1.000000 \\
\hline
\end{tabular}


Table 5.

Correlation of morphometric variables with body length in male Echinorhynchus truttae. Correlation measured by Pearson's product-moment correlation coefficient $(r)$. The raw $p$ value is the probability that the sample correlation coefficient could have come from a population with a correlation coefficient of zero. The Bonferroni correction was used to control the family wise error rate across multiple tests of significance. Data available in Suppl. material 2.

\begin{tabular}{|l|l|l|l|l|}
\hline Variable & $\mathbf{n}$ & $\mathbf{r}$ & raw $\mathbf{~}$ & Bonferroni p \\
\hline Reproductive system length & 32 & 0.936 & $<0.000001$ & $<\mathbf{0 0 0 0 0 1}$ \\
\hline Body width & 32 & 0.417 & 0.017468 & 0.314424 \\
\hline Proboscis length & 32 & 0.298 & 0.097440 & 1.000000 \\
\hline Proboscis width & 32 & -0.054 & 0.769724 & 1.000000 \\
\hline Proboscis receptacle length & 32 & 0.131 & 0.474205 & 1.000000 \\
\hline Proboscis receptacle width & 32 & 0.236 & 0.193402 & 1.000000 \\
\hline Lemniscus length & 32 & 0.698 & 0.000009 & $\mathbf{0 . 0 0 0 1 5 9}$ \\
\hline Lemniscus width & 32 & 0.330 & 0.064692 & 1.000000 \\
\hline Anterior testis length & 28 & 0.588 & 0.001008 & $\mathbf{0 . 0 1 8 1 5 2}$ \\
\hline Anterior testis width & 28 & 0.446 & 0.017358 & 0.312447 \\
\hline Posterior testis length & 28 & 0.685 & 0.000059 & $\mathbf{0 . 0 0 1 0 5 8}$ \\
\hline Posterior testis width & 28 & 0.352 & 0.065541 & 1.000000 \\
\hline Cement gland width & 32 & 0.296 & 0.099633 & 1.000000 \\
\hline Saefftigen's pouch length & 32 & 0.360 & 0.043181 & 0.777265 \\
\hline Saefftigen's pouch width & 32 & 0.174 & 0.339571 & 1.000000 \\
\hline Penis width & 32 & 0.217 & 0.232671 & 1.000000 \\
\hline Bursal sucker diameter & 15 & 0.259 & 0.350967 & 1.000000 \\
\hline Maximum length of hook blade & 23 & 0.428 & 0.041548 & 0.747868 \\
\hline
\end{tabular}




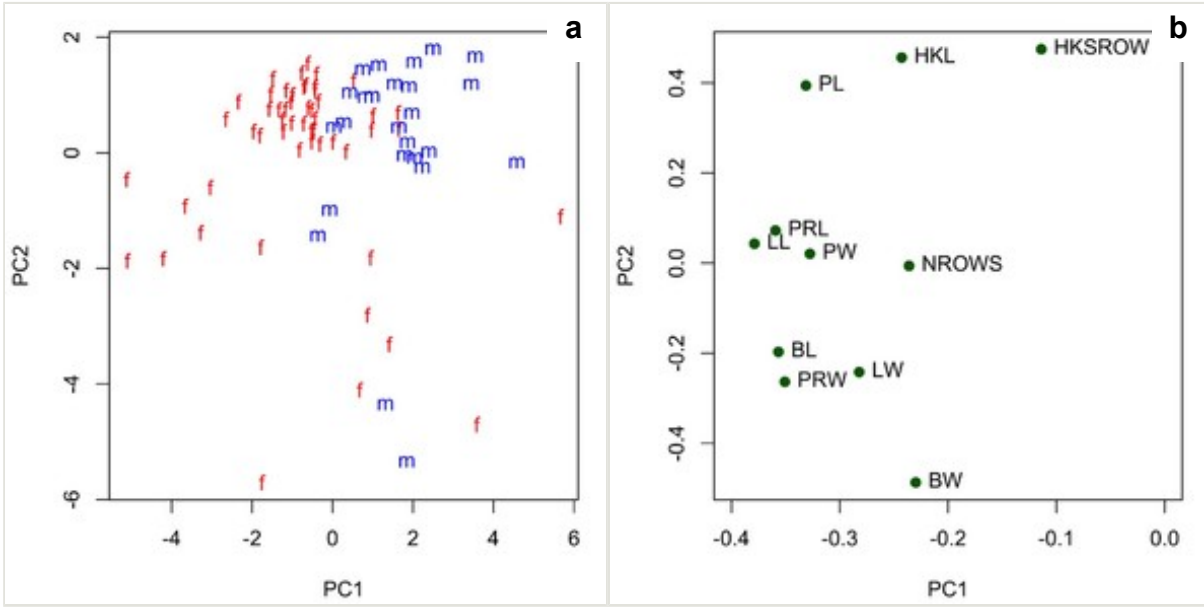

Figure 1.

Sexual dimorphism in Echinorhynchus truttae revealed by principal component analysis of morphometric and meristic variables for 53 females and 25 males. The first (PC1) and second (PC2) principal components account for $36 \%$ and $24 \%$ of the variation in the data, respectively. Analysis based on data in Suppl. materials 1, 2.

a: Scatterplot of the scores for the first two principal components (PC1 and PC2). Key: f, female; m, male.

b: Scatterplot of the loadings for PC1 and PC2. Key: BL, body length; BW, body width; PL, proboscis length; PW, proboscis width; PRL, proboscis receptacle length; PRW, proboscis receptacle width; LL, lemniscus length; LW, lemniscus width; HKL, maximum hook blade length; NROWS, number of longitudinal rows of hooks; HKSROW, maximum number of hooks per longitudinal row.

Although there are interspecific differences in the means of some of the morphometric variables (e.g. maximum length of hook blade) listed in Tables 2, 3, interspecific overlap in their ranges prevents any single morphometric variable from being used to reliably discriminate any of the species in this study. For a graphical representation of interspecific variation in each conventional morphological character, see boxplots in Suppl. materials 9, 10.

Marked intraspecific, but subtle interspecific anatomic variation was observed in the male reproductive system. Four of 32 male $E$. truttae had only one testis, which measured 793$1530 \times 393-730 \mu \mathrm{m}$. No monorchid males were found in E. bothniensis or E. 'bothniensis'. All of the Echinorhynchus spp. studied typically displayed six cement glands, but the number of glands was variable in $E$. 'bothniensis' and $E$. truttae. Of eleven specimens of $E$. 'bothniensis', nine (82\%) exhibited six cement glands, but two (18\%) had only five. Cement gland number was recorded from 35 male E. truttae; the numbers displaying 4, 5, 6 and 8 cement glands were $1(3 \%), 3(9 \%), 30(86 \%)$ and $1(3 \%)$, respectively. Cement gland arrangements of specimens with six glands are summarized in Table 6 . It is interesting to note that none of the specimens of $E$. truttae were found to exhibit the moniliform pattern (chain-like, six singles) and that the majority (96\%) had either one or two paired cement 
glands. This is in contrast to the other taxa, where a large proportion of the males $(21-57 \% \square$ display the moniliform pattern. In E. 'bothniensis' pairs of cement glands consisted of the third and fourth, or fourth and fifth glands from the anterior. In E. bothniensis pairs were made up of any two adjacent cement glands (i.e. first and second, second and third, third and fourth, fourth and fifth or fifth and sixth).

Table 6.

Cement gland arrangement in males of the Echinorhynchus bothniensis group and $E$. truttae

Notation for cement gland pattern from Shostak et al. (1986): B, clumped, three staggered pairs; C, chainlike, two pairs and two singles; D, chainlike, one pair and four singles; E, chainlike, six singles. Only specimens with six cement glands included. Data available in Suppl. material 2.

\begin{tabular}{|l|l|l|l|l|}
\hline & B & C & D & E \\
\hline E.bothniensis (Lake Keitele) & 1 & 4 & 10 & 4 \\
\hline E. 'bothniensis' (Lake Pulmankijärvi) & $(5.30 \%)$ & $(21.10 \%)$ & $(52.60 \%)$ & $(21.10 \%)$ \\
\hline E.leidyi (Northern Canada, Shostak et al., 1986) & 0 & 0 & 4 & 5 \\
\hline E.truttae (Scotland) & & & $(44.40 \%)$ & $(55.60 \%)$ \\
\hline & 1 & 36 & 181 & 118 \\
\hline & $(0.30 \%)$ & $(10.70 \%)$ & $(53.90 \%)$ & $(35.10 \%)$ \\
\hline & 1 & 16 & 13 & 0 \\
\hline
\end{tabular}

\section{Proboscis profiles}

Before attempting to use the Proboscis Profiler to discriminate taxa, potential confounding variables should be considered. Preparation is one such problem (Palaearctic samples fixed in alchol, then cleared and temporarily mounted in lactophenol vs Nearctic samples fixed in FAA, stained with acetocarmine and permanently mounted in synthentic resin), but cannot be controlled in this analysis. Therefore, it is important to exercise caution when making comparisons between E. leidyi and the other taxa. Radial asymmetry of proboscis hooks is another potential problem (Wayland 2010). Unfortunately, the importance of radial asymmetry was not known at the time of data collection and so no record was made of which surface of the proboscis (dorsal, ventral or lateral) the measured hooks were situated. One confounding factor which can be measured and, if necessary, controlled (by profiling females and males separately) is sexual dimorphism. This phenomenon was investigated in $E$. truttae, because hook data from a complete longitudinal row are available (Suppl. materials 4,5$)$ for a relatively large number of both female $(n=46)$ and male $(n=26)$ acanthocephalans.

Fig. 2 shows hook blade length and base width variables of the 72 E. truttae specimens plotted against a standardized position (for definition, see morphological data analysis section of material and methods). Sexual dimorphism is not readily apparent in these two plots. Proboscis profiles were generated with a moving average segment of 11 ; the minimum sized moving average segment that can be applied to this dataset. Principal 
component analysis of these proboscis profiles revealed subtle sexual dimorphism, with some separation of the females from males in principal component one (PC1), which describes $49 \%$ of the variation in the dataset (Fig. 3a). A Welch two sample t-test found a significant difference $(p=0.005)$ between females and males in the scores for PC1. Base width variables show higher loadings than blade length variables for PC1 (Fig. 3b), suggesting that female $E$. truttae tend to have 'stouter' hooks than males. In view of this strong evidence of sexual dimorphism in proboscis profiles, the two sexes are considered separately in the inter-specific comparisons that follow.
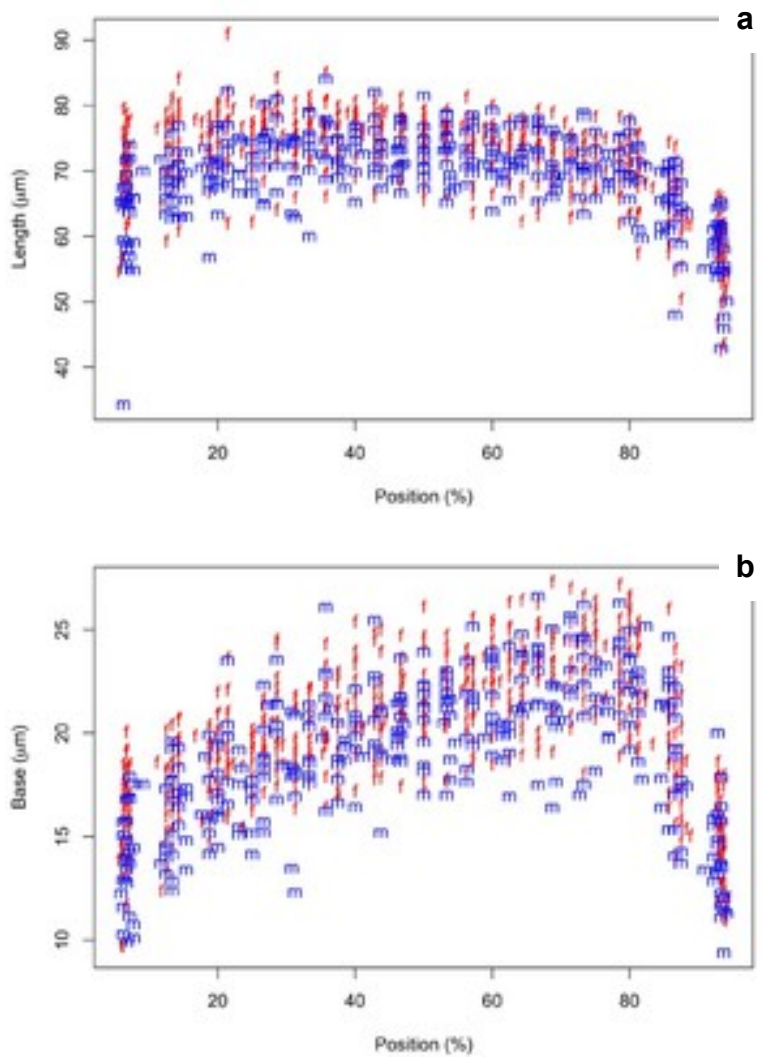

Figure 2.

Positional variation in two hook morphometrics of female and male Echinorhynchus truttae (number of individuals are 46 and 26 respectively). Analysis based on data in Suppl. materials 4, 5.

a: Hook blade length plotted against standardized position.

b: Hook base width plotted against standardized position. 


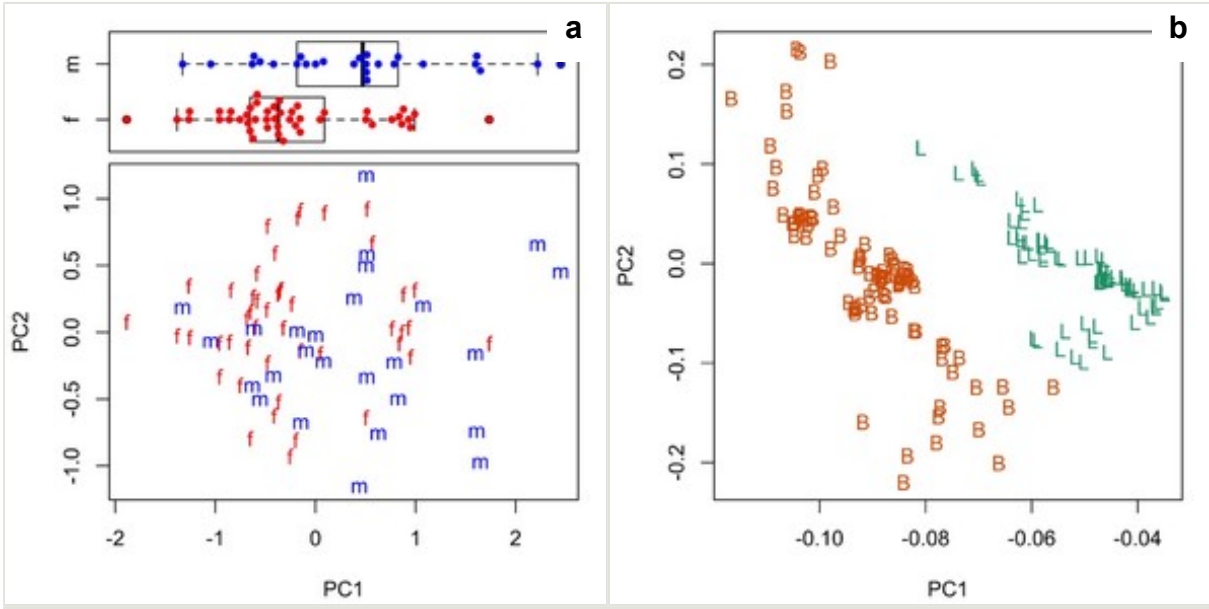

Figure 3.

Principal component analysis of the proboscis profiles of female and male Echinorhynchus truttae. The first (PC1) and second (PC2) principal components describe $49 \%$ and $15 \%$ of the variance in the data. Analysis based on data in Suppl. materials 4, 5 .

a: Scatterplot of the scores for the first two principal components (PC1 and PC2). Inset boxplot shows distribution of scores for PC1. Key: $f$, female; $m$, male.

b: Scatterplot of the loadings for PC1 and PC2. Key: L, length variables; B, base variables.

Proboscis profiles for 56 female acanthocephalans (5 of $E$. bothniensis, 2 of $E$. 'bothniensis', 3 of $E$. leidyi and 46 of $E$. truttae) were generated using a moving average segment of 10; the minimum sized moving average segment applicable. This dataset of female hook morphometrics (Suppl. material 4) includes data from one of the paratypes of E. bothniensis from the Bothnian Bay. Fig. 4 shows positional variation in raw hook morphometrics of female worms; whilst some interspecific variation is apparent, the taxa are indistinguishable. A principal component analysis of the proboscis profiles was performed and a scatterplot of the scores for the first two principal components (Fig. 5a) shows a clear separation of $E$. truttae from the other taxa. The loadings plot for the first two principal components (Fig. 5b) shows that blade length and base width measurements from hooks in the $80.5-95.5 \%$ region of the proboscis are driving the separation of $E$. truttae from the other taxa along PC1 (this first principal component accounts for $64 \%$ of the variance in the dataset). E. bothniensis, E. 'bothniensis' and E. leidyi are not separated from each other in the scores plot for PC1 and PC2. Hierarchical clustering was used to objectively partition the proboscis profiles into morphotypes; a Euclidean distance matrix was calculated from the scores for PC1 and PC2 and a dendrogram was computed using the complete agglomeration method as implemented in the $\underline{R}$ function hclust (Fig. 6). The dendrogram shows the presence of two distinct groups: one containing all profiles of $E$. truttae and the other comprising the profiles of the other taxa. The proboscis profile of one specimen of $E$. leidyi clustered with the $E$. truttae profiles. The E. truttae cluster comprises two subclusters which are not related to geographical location. 

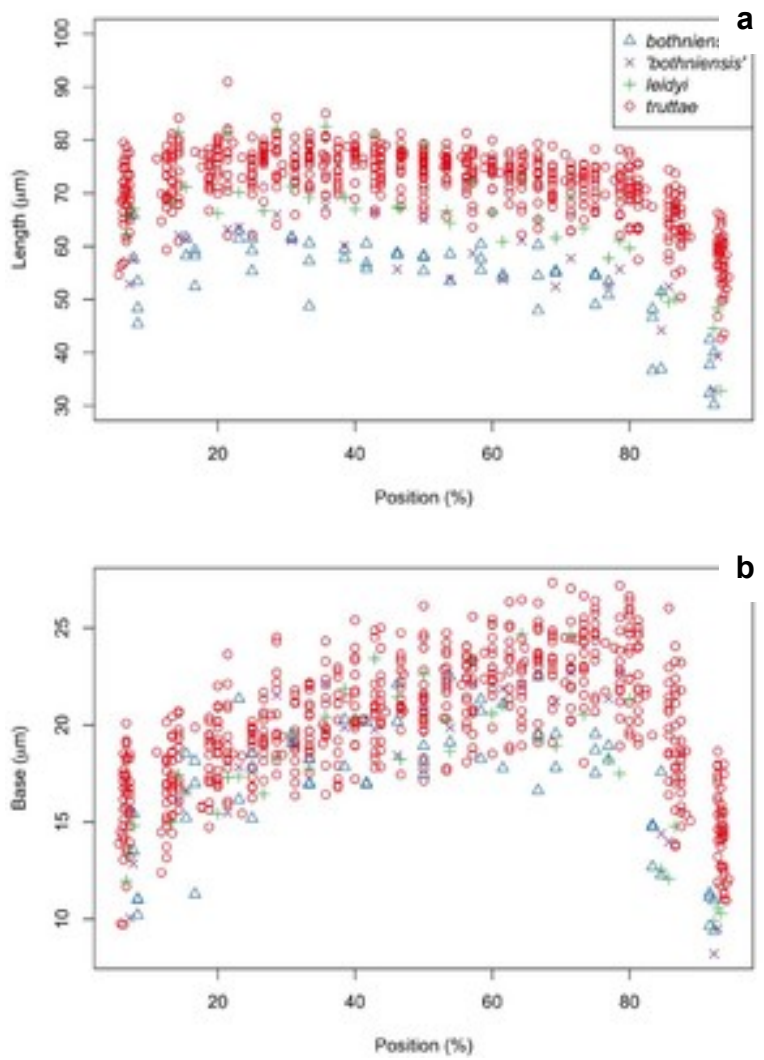

Figure 4.

Positional variation in two hook morphometrics of female Echinorhynchus bothniensis, $E$. 'bothniensis', E. leidyi and E. truttae (number of individuals were 5, 2, 3 and 46, respectively). Analysis based on data in Suppl. material 4.

a: Hook blade length plotted against standardized position.

b: Hook base width plotted against standardized position. 

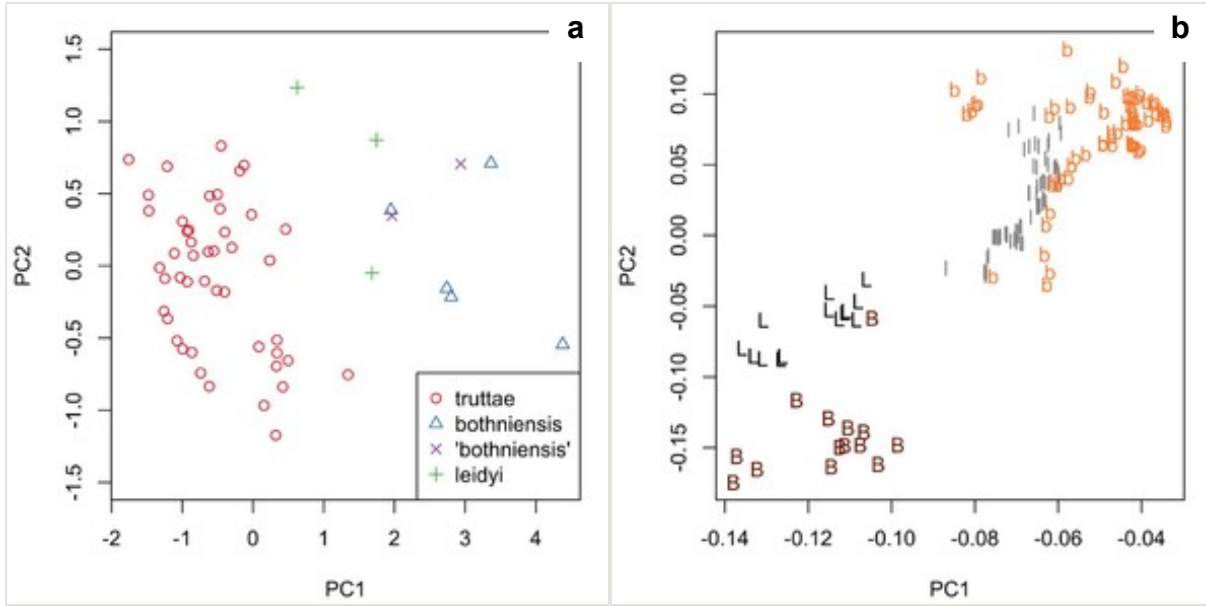

Figure 5.

Principal component analysis of the proboscis profiles of female Echinorhynchus bothniensis, $E$. 'bothniensis', E. leidyi and E. truttae. The first (PC1) and second (PC2) principal components describe $64 \%$ and $10 \%$ of the variance in the data, respectively. Analysis based on data in Suppl. material 4.

a: Scatterplot of the scores for the first two principal components (PC1 and PC2).

b: Scatterplot of the loadings for the first two principal components. Key: I and b, length and base measurements respectively, from hooks in the $4.5-79.5 \%$ region of the proboscis; $L$ and $B$, length and base measurements respectively, from hooks in the $80.5-95.5 \%$ region of the proboscis.

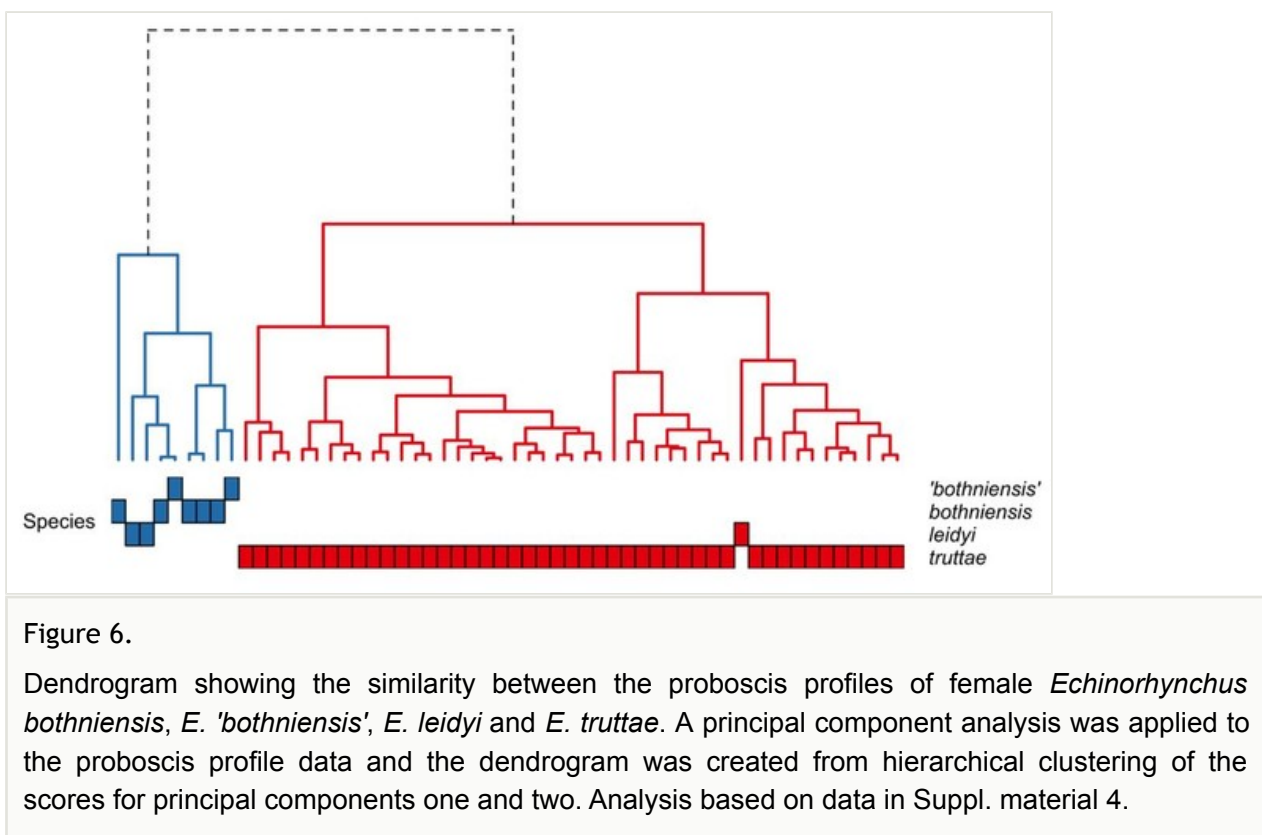


None of the male specimens of E. 'bothniensis' had fully everted proboscides and so hook morphometric data could not be collected from them. Therefore, the analysis of interspecific variation in proboscis profiles for male worms was limited to three species: $E$. bothniensis $(\mathrm{n}=5)$, E. leidyi $(\mathrm{n}=10)$ and E. truttae $(\mathrm{n}=26)$ (data available as Suppl. material 5). Plots of hook morphometrics against standardized position (Fig. 7) show some separation of $E$. truttae from the other taxa; this is most apparent in blade length measurements towards the base of the proboscis (Fig. 7b). Proboscis profiles were generated with a moving average segment of 11 , the minimum applicable to the dataset, and then further investigated using principal components analysis. A scores plot for PC1 and PC2 (Fig. 8a) showed a clear separation of E. truttae from the other two taxa, and a partial separation of $E$. bothniensis from E. leidyi. As was found for the female proboscis profiles, blade length and base width measurements from hooks at the base of the proboscis (80-95\% region) are driving the separation of $E$. truttae from the other taxa (Fig. 8b). Hierarchical clustering partioned the male proboscis profiles into three groups corresponding to the three taxa (Fig. 9). However, the proboscis profiles for one of the 10 speciemens of $E$. leidyi was placed in the $E$. bothniensis cluster. As in the dendrogram for female acanthocephalans, the $E$. truttae branch bifurcates into two subclusters which are not related to sampling locality. 

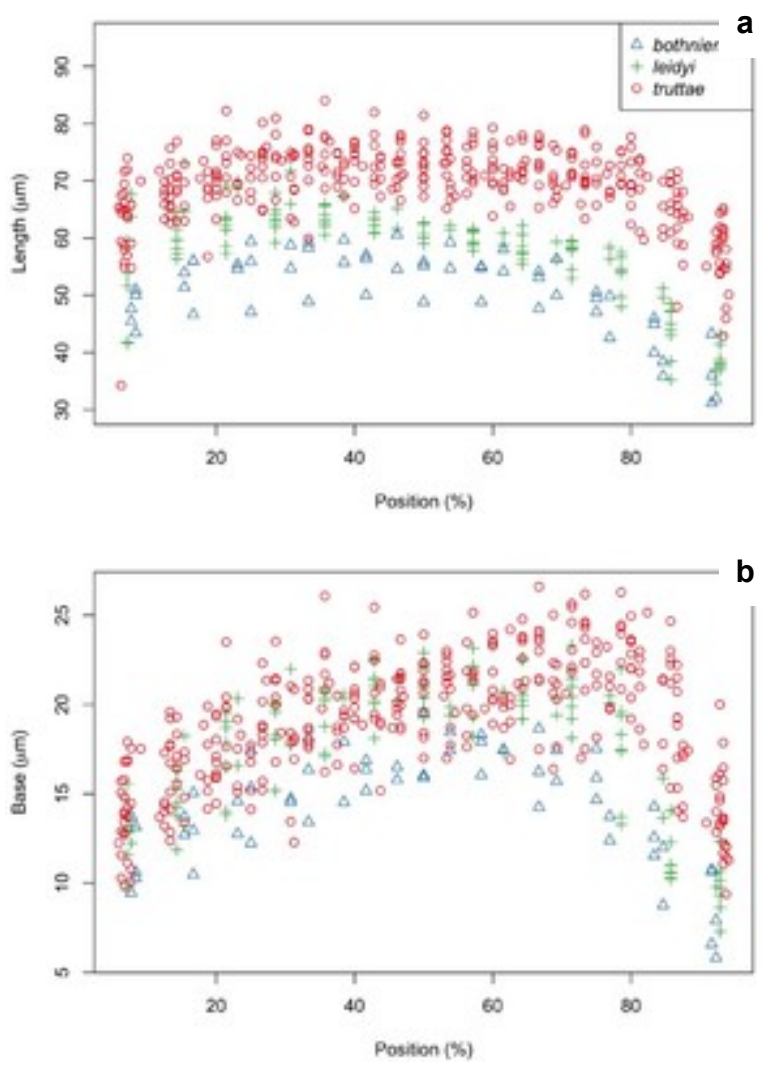

Figure 7.

Positional variation in two hook morphometrics of male Echinorhynchus bothniensis, E. leidyi and E. truttae (number of individuals are 5, 10 and 26 respectively). Analysis based on data in Suppl. material 5.

a: Hook blade length plotted against standardized position.

b: Hook base width plotted against standardized position. 

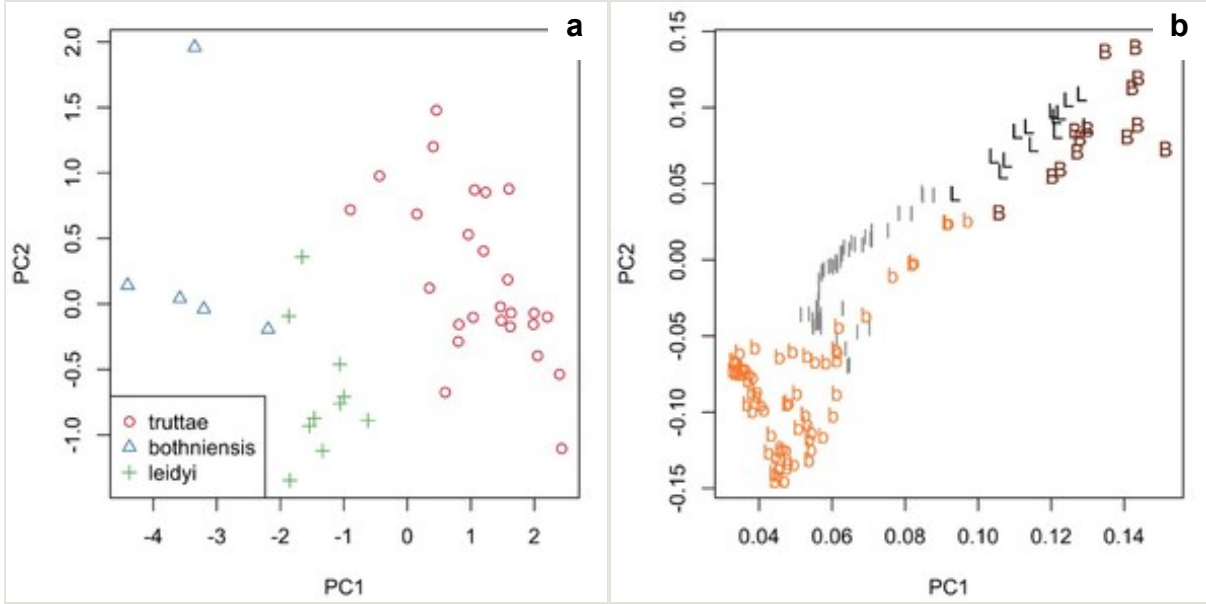

Figure 8.

Principal component analysis of the proboscis profiles of male Echinorhynchus bothniensis, $E$. leidyi and E. truttae. The first (PC1) and second (PC2) principal components describe $70 \%$ and $12 \%$ of the variance in the data respectively. Analysis based on data in Suppl. material 5.

a: Scatterplot of the scores for the first two principal components (PC1 and PC2).

b: Scatterplot of the loadings for the first two principal components. Key: I and b, length and base measurements respectively, from hooks in the $5-79 \%$ region of the proboscis; $L$ and $B$, length and base measurements respectively, from hooks in the $80-95 \%$ region of the proboscis.

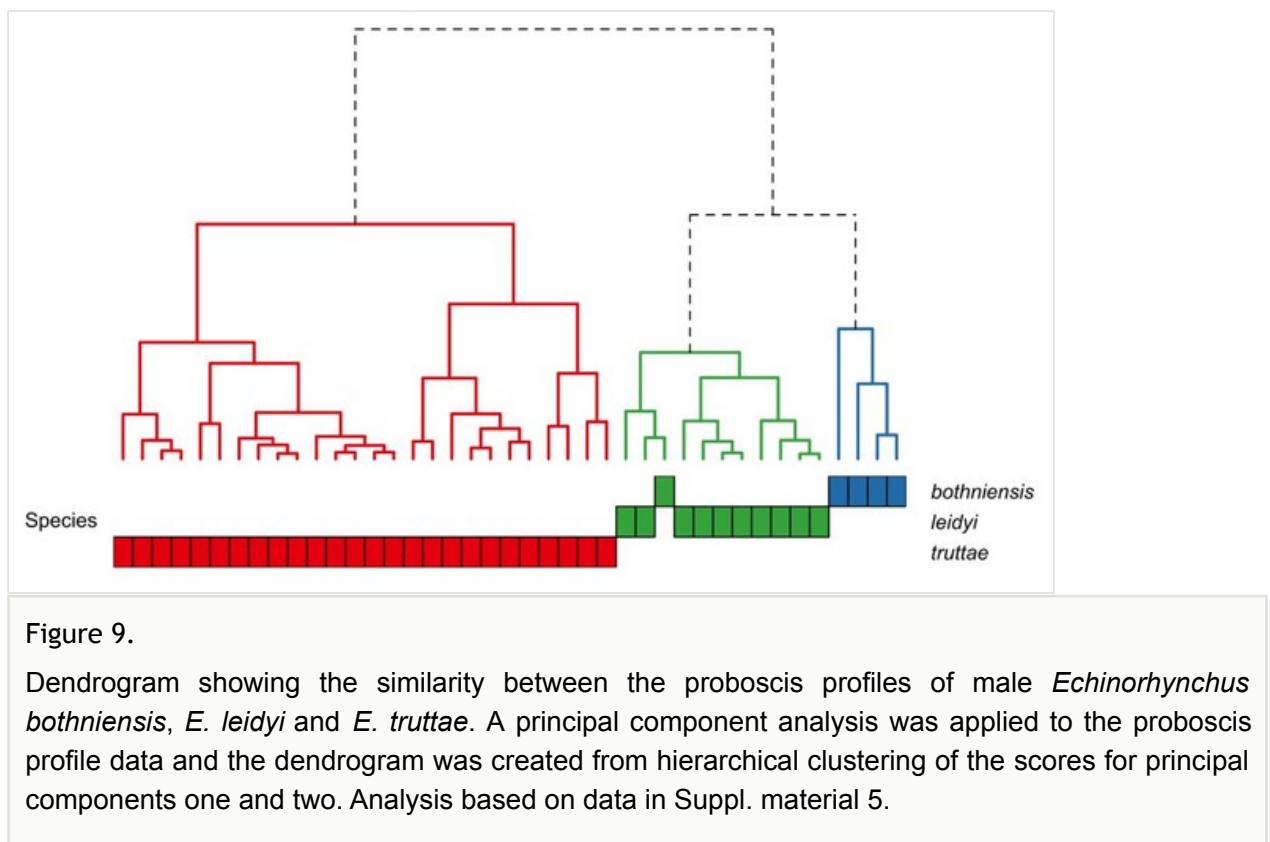




\section{Ecological observations}

The frequency distribution of $E$. truttae in its definitive host Salmo trutta was recorded for two localities: Loch Walton Burn and Loch Coulter Burn (summary statistics in Table 7; raw data available in Suppl. materials 6,7). Prevalence of infection was low in both host populations, as were the mean and maximum intensity of infection. Nevertheless, the acanthocephalans were successfully mating, as evident from the presence of gravid females in fish from both localities. The aggregation index was greater than unity in both localities, indicating that the acanthocephalans were overdispersed in their host populations. To further investigate the frequency distribution of the parasite in its host populations, two theoretical distributions were fitted to each dataset (Fig. 10); the Poisson distribution is a good model for a random distribution, while the negative binomial describes overdispersion. A chi-squared test showed that a fitted negative binomial distribution was not significantly different from the observed distribution at both localities (Loch Walton, chi-squared statistic 2.03, p-value 0.155; Loch Coulter, chi-squared statistic 1.81, $p$-value 0.178). Conversely, the Poisson distribution was a poor fit to the observed data (Loch Walton, chi-squared statistic 13.2, p-value 0.00135; Loch Coulter, chi-squared statistic 6.13, p-value 0.0467).

Table 7.

Frequency distribution of Echinorhynchus truttae in definitive host populations. $95 \%$ confidence limits (where applicable) in parentheses. Data available in Suppl. materials 6, 7.

\begin{tabular}{|l|l|l|}
\hline & Loch Coulter Burn & Loch Walton Burn \\
\hline Number of fish examined & 42 & 46 \\
\hline Prevalence (\%) & $0.119(0.048-0.259)$ & $0.283(0.171-0.434)$ \\
\hline Mean intensity of infection & $1.4(1.0-1.6)$ & $2(1.46-2.69)$ \\
\hline Maximum intensity of infection & 2 & 5 \\
\hline Mean abundance & $0.167(0.0476-0.333)$ & $0.565(0304-0.935)$ \\
\hline Overdispersion index (variance/mean) & 1.44 & 2.1 \\
\hline
\end{tabular}




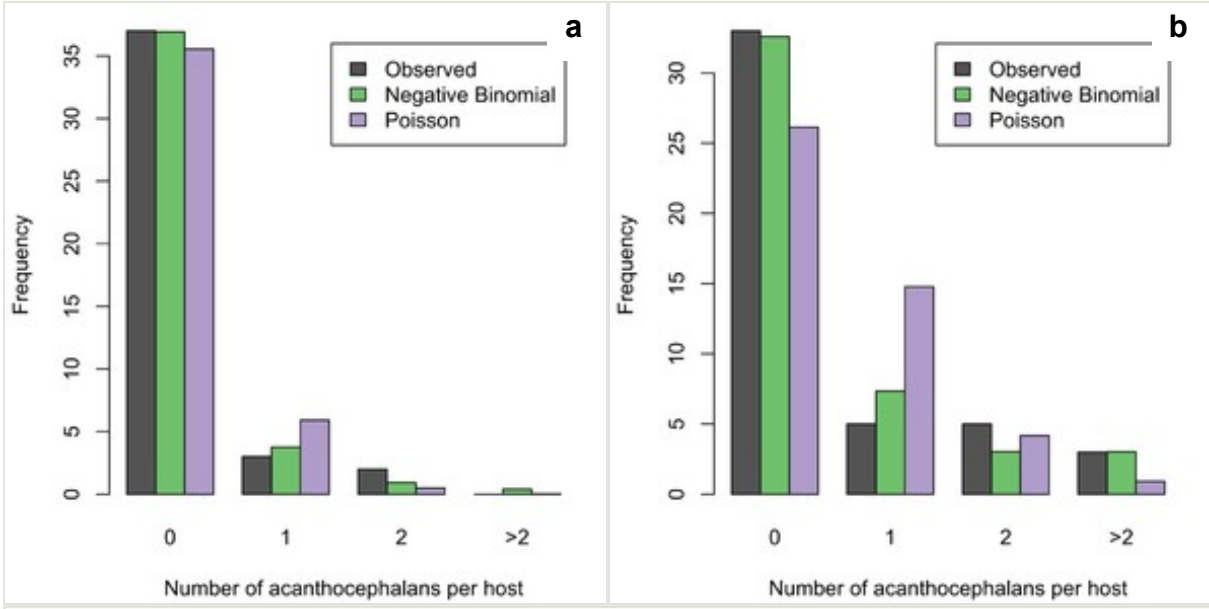

Figure 10.

Observed and fitted distributions of Echinorhynchus truttae in two populations of its definitive host Salmo trutta. Analysis based on data in Suppl. materials 6, 7.

a: Loch Coulter Burn. Negative binomial distribution has parameters: mu=0.167 and k=0.261. Poisson distribution has parameter lambda=0.167. Akaike's information criterion (AIC) for fitted distributions: negative binomial, 43.3; Poisson, 43.9.

b: Loch Walton Burn. Negative binomial distribution has parameters: mu=0.565 and k=0.375. Poisson distribution has parameter lambda=0.565. AIC for fitted distributions: negative binomial, 97.5; Poission, 107.3.

G. pulex, the intermediate host of E. truttae, was abundant in both streams. One hundred specimens of this amphipod from Loch Walton Burn were examined by dissection, and while no larval E. truttae were found, four cystacanths of Polymorphus minutus (Goeze, 1782) (Polymorphida: Polymorphidae) were encountered.

\section{Discussion}

\section{Intraspecific morphological variation}

This study provides the first detailed account of morphometric and meristic variation in adult $E$. truttae, albeit for populations within a small part of its known geographical range. In the absence of evidence to the contrary, the $E$. truttae samples are assumed to comprise a single biological species. However, given the ubiquity of cryptic speciation in the Acanthocephala (Buron et al. 1986, Väinölä et al. 1994, Steinauer et al. 2007, Martínez-Aquino et al. 2009), this assumption might be unwarranted. The E. truttae material examined in the present study conforms well to other published descriptions (Lühe 1911, Meyer 1933, Hoffman 1954) but displays considerably greater morphological variability. The only notable difference between the descriptions provided by different authors concerns the size of the eggs. The wide range of egg dimensions recorded in the present study $(120-173 \times 22-34 \mu \mathrm{m})$ ecompasses the measurements reported by Hoffman 
(1954) $(138 \times 24 \mu \mathrm{m})$, but not the range of dimensions reported by Lühe $(1911)(100-110 \times$ 23-24 $\mu \mathrm{m})$ and Meyer (1933) $(100-110 \times 24 \mu \mathrm{m})$. Discrepancies in egg dimensions between different studies are most likely the result of different fixatives and clearing agents being used to prepare the material for light microscopy, but may also be due to differences in the state of maturity of the acanthors. Shrinkage of eggs following fixation, staining and mounting has been reported by many authors (e.g. Lynch 1936, Cleave and Timmons 1952, Cable and Hopp 1954, Bullock 1962).

E. truttae exhibited sexual dimorphism in all morphometric variables common to both genders. Within each gender, a proportion of the variance in some morphometric variables was explained by body length. Seven morphometric variables (body width, proboscis length, proboscis receptacle length, lemniscus length and width, vagina width and vaginal sphincter width) were found to be positively correlated with body length in female worms, whilst just four (length of reproductive system, lemniscus length, length of both anterior and posterior testis) showed this relationship in males. However, the length range and sample size of male worms was small relative to that of females and this would have made it more difficult to find evidence of any correlation. A positive correlation with body length can be demonstrated for the size of most anatomical structures in palaecanthocephalans (e.g. Amin and Redlin 1980, Brown 1987). Awachie (1966) found that both female and male $E$. truttae increase in length with time spent in the intestine of their definitive host, S. trutta, and that proboscis length increases with body size. Furthermore, body length and time spent in the definitive host intestine were also positively correlated with sexual maturation in female worms.

Proboscis profiler provided tentative evidence for the presence of two distinct morphotypes within E. truttae (Figs 6, 9). This variation was not related to geography, as both subgroups contained samples from both the River Carron catchment, central Scotland and Drummore, southwest Scotland. A molecular genetic analysis would be required to test the hypothesis that these two apparent morphotypes represent sibling species.

Small sample sizes prohibited a statistical analysis of intraspecific morphological variation in the other taxa studied. However, comparison of the mean values and ranges of most morphometric variables (Tables 2, 3) suggest that these taxa also display sexual dimorphism. The Bothnian Bay and Lake Keitele populations of $E$. bothniensis are thought to have been reproductively isolated for at least 6000 years (Väinölä et al. 1994); however, this study did not find any obvious morphological divergence between them.

\section{Discrimination of species using morphological characters}

The genetic differentiation of $E$. bothniensis and $E$. 'bothniensis' into distinct biological species, as evidenced from allozyme electrophoresis (Väinölä et al. 1994), was not accompanied by obvious divergence in conventional morphological characters. Furthermore, proboscis profiler failed to discriminate these species on the basis of female hook morphometrics. Proboscis profiler could not be used to compare the males of these species, as hook data were not available for male E. 'bothniensis'. Proboscis profiler has been used to successfully discriminate two species of the $E$. gadi species group identified 
by allozyme electrophoresis (Wayland 2010). However, E. bothniensis and E. 'bothniensis' probably diverged more recently than the sibling species of the $E$. gadi group (Väinölä et al. 1994) and therefore have had less time to undergo adaptive morphological change. Moreover, if E. bothniensis and E. 'bothniensis' occur in allopatry, but utlise similar intermediate and definitive hosts, there may be little or no selection pressure to drive morphological divergence. In contrast, the sibling species of $E$. gadi separable by Proboscis profiler occur in sympatry and often in the same host individual. In this case, adaptation to different regions of the definitive host intestine to avoid competition and/or hybridization may have resulted in anatomical changes to the hooks of the proboscis (Wayland et al. 2005).

The anatomically similar E. leidyi from the Nearctic has not been investigated using molecular markers and so its systematic homogeneity and relationship to $E$. bothniensis and $E$. 'bothniensis' may only be speculated. E. leidyi could not be discriminated from $E$. bothniensis or E. 'bothniensis' using any conventional morphological character or the proboscis profiles of female worms. When applied to male worms, proboscis profiler was quite successful in separating four specimens of $E$. bothniensis from ten specimens of $E$. leidyi, however a fifth specimen of $E$. bothniensis was assigned to the E. leidyi cluster (Fig. 9). Nevertheless, this observation should be interpreted with caution as it is based on a small sample of acanthocephalans and may be an artifact of the different protocols used to prepare samples of the two taxa for light microscopy.

The inability of multivariate statistical analysis to reliably distinguish the Nearctic E. leidyi from the Palaearctic E. bothniensis and E. 'bothniensis', on the basis of morphological characters, is further evidence of the phylogenetic affinity of these taxa. If these acanthocephalans have co-speciated with their mysid intermediate hosts, as hypothesised by Väinölä et al. (1994), they will be members of a clade comprising at least four sibling species (Audzijonytė and Väinölä 2005), some of which may occur in sympatry and at least one may have a circumarctic distribution. An extensive sampling effort combined with tandem molecular and morphological analysis was needed to differentiate and characterize the species of the $M$. relicta (sensu lato) group; a similar strategy will be required to investigate the diversity in their echinorhynchid parasites.

E. truttae could not be discriminated from E. leidyi and the $E$. bothniensis species complex on the basis of any single conventional morphological character. However, Proboscis profiler successfully separated E. truttae from E. leidyi, E. bothniensis and E. 'bothniensis'. The hook morphometric data available here as supplementary files (Suppl. materials 4,5 ) serve as a useful reference for $E$. truttae, E. leidyi and the $E$. bothniensis species group, to which new samples of Echinorhynchus spp. from fresh and brackish waters can be compared using Proboscis profiler.

\section{Distribution of acanthocephalans in their definitive host populations}

The frequency distribution of macroparasites within their host populations almost invariably shows overdispersion or aggregation; most hosts harbour few or no parasites, and a few hosts harbour large numbers of parasites (Crofton 1971, Pennycuick 1971, Anderson and 
May 1978, Anderson and Gordon 1982, Dietz 1982, Dobson 1985, Grenfell et al. 1986, Pacala and Dobson 1988, Guyatt and Bundy 1991, Shaw et al. 1998). Overdispersion is described empirically by the negative binomial distribution (Crofton 1971). In the case of natural infections of Acanthocephala, this distribution has previously been shown to provide an accurate description of the following species in their definitive host populations: Acanthocephalus clavula (Dujardin, 1845) in Gasterosteus aculeatus L. (see Pennycuick 1971) and Anguilla anguilla (L.) (see Shaw et al. 1998); Acanthocephalus lucii (Müller, 1776) in Perca fluviatilis (L.) (see Shaw et al. 1998); and Echinorhynchus canyonensis Huffman \& Kliever, 1977 in Maynea californica Gilbert (see Huffman and Kliever 1977). In this study the negative binomial provided a good model of the distribution of $E$. truttae in two populations of its definitive host S. trutta. However, Hine and Kennedy (1974) found that the negative binomial was a poor fit to the frequency distribution of Pomphorhynchus laevis (Müller, 1776) in Leuciscus leuciscus (L.), even though the parasite was not randomly distributed in its host population.

The negative binomial distribution has also been used to quantify aggregation of larval acanthocephalans in populations of their intermediate hosts. Hine and Kennedy (1974) found that it was a good fit to the observed frequency distribution of $P$. laevis in a population of Gammarus pulex (L.). If there is parasite-induced host mortality, as in the case of natural infections of G. pulex by Polymorphus minutus (Goeze, 1782), then a truncated negative binomial model is more appropriate (Crofton 1971).

Overdispersion of parasites in their host populations may have various causes, including seasonality in the occurrence of infective stages, spatial aggregation of infective stages, and differences between hosts in behaviour, physiology and immune response to the parasites (e.g. Crofton 1971, Pacala and Dobson 1988, Shaw et al. 1998). E. truttae is known to display a seasonal pattern of abundance in its intermediate host, G. pulex (see Awachie 1966). However, seasonality should only be a cause of overdispersion in datasets comprising samples taken throughout the year; in this study the two E. truttae datasets each represented single samples.

Aggregation of cystacanths of E. truttae in its amphipod intermediate host G. pulex, is a potential cause of the acanthocephalan's overdispersion in its definitive host $S$. trutta. Since cystacanths of $P$. minutus and $P$. laevis have been found to be aggregated in populations of G. pulex, then it is plausible that the same phenomenon occurs in E. truttae. If the larvae of $E$. truttae were aggregated in their intermediate host population, then, although their fish hosts may have encountered intermediate hosts at random, the worm burden of the intermediate hosts encountered would not be random. This would lead to a heterogenous distribution of acanthocephalans in the fish population.

It is important to note that overdispersion of acanthocephalans in their definitive hosts can occur in the absence of spatial aggregation of cystacanths. Crompton et al. (1984) found that Moniliformis moniliformis (Bremser, 1811) Travassos, 1915 (as Moniliformis dubius Meyer, 1932) had an aggregated distribution in groups of rats (Rattus norvegicus (Berkenhout)) in which every rat had been fed the same number of cystacanths. Valtonen and Crompton (1990) found that the prevalence and overdispersion of E. bothniensis 
infections of $O$. eperlanus increased with host size. This observation suggests that overdispersion in this particular host-parasite system is linked to some aspect of the interaction between parasite and definitive host.

Experimental work is necessary to determine the causes of overdispersion of acanthocephalans in their host populations. Moniliformis moniliformis in rats serves as a convenient laboratory model for studies on acanthocephalan dispersion in mammalian host populations (Crompton et al. 1984, Stoddart et al. 1991). E. truttae in S. trutta might represent a useful model for studies of acanthocephalan dispersion in fish populations, since this species has a life cycle which can be completed in the laboratory (Awachie 1966).

\section{Acknowledgements}

The author would like to thank Professor Tellervo Valtonen (University of Jyväskylä, Finland) and Dr Alan Pike (University of Aberdeen, UK) for collecting most of the samples used in this study. The Canadian Museum of Nature kindly lent the specimens of E. leidyi. The author would also like to thank the reviewers, Dr David Gibson and Dr Plamen Pankov, for their insightful comments which substantially improved the quality of this paper.

\section{References}

- Amin O, Redlin M (1980) The effect of host species on growth and variability of Echinorhynchus salmonis Müller, 1784 (Acanthocephala: Echinorhynchidae), with special reference to the status of the genus. Systematic Parasitology 2 (1): 9-20. DOI: 10.1007/BF00015091

- Anderson R, Gordon D (1982) Processes influencing the distribution of parasite numbers within host populations and special emphasis on parasite-induced host mortalities. Parasitology 85 : 373-398. DOI: 10.1017/S0031182000055347

- Anderson R, May R (1978) Regulation and stability of host-parasite population interactions-I. Regulatory processes. Journal of Animal Ecology 47: 219-247. DOI: 10.2307/3933

- Audzijonytė A, Väinölä R (2005) Diversity and distributions of circumpolar fresh- and brackishwater Mysis (Crustacea: Mysida): descriptions of M. relicta Lovén, 1862, M. salemaai n. sp., M. segerstralei n. sp. and M. diluviana n. sp., based on molecular and morphological characters. Hydrobiologia 544 (1): 89-141. DOI: 10.1007/s10750-004-8337-7

- Awachie J (1966) The development and life history of Echinorhynchus truttae Schrank 1788 (Acanthocephala). Journal of Helminthology 40: 11-32. DOI: 10.1017/S0022149X00034040

- Baldanova D (2000) A fecundity of proboscis worms of the genus Echinorhynchus (Acanthocephala: Echinorhynchidae) from Baikal Lake. Parazitologiya, Akademiya Nauk, SSSR, Leningrad 34 (2): 150-153. [In Russian].

- Baldanova D, Pronin N (1998) The seasonal dynamics of infection of salmonids with Echinorhynchus salmonis and E. truttae (Acanthocephala: Echinorhynchidae) in the Baikal Lake. Parazitologiya, Akademiya Nauk SSSR, Leningrad 32 (1): 71-78.

- Brown A (1987) Anatomical variability and secondary sexual characteristics in Pomphorhynchus laevis (Müller, 1776) (Acanthocephala). Systematic Parasitology 9 (3): 213-219. DOI: 10.1007/ BF00010856 
- Bullock W (1962) A New Species of Acanthocephalus from New England Fishes, with Observations on Variability. The Journal of Parasitology 48 (3): 442. DOI: $10.2307 / 3275212$

- Buron I, Renaud F, Euzet L (1986) Speciation and specificity of acanthocephalans. Genetic and morphological studies of Acanthocephaloides geneticus sp. nov. parasitizing Arnoglossus laterna (Bothidae) from the Mediterranean littoral (Sète-France). Parasitology 92 (1): 165. DOI: $10.1017 /$ S0031182000063526

- Cable R, Hopp W (1954) Acanthocephalan Parasites of the Genus Neoechinorhynchus in North American Turtles with the Descriptions of Two New Species. The Journal of Parasitology 40 (6): 674. DOI: $10.2307 / 3273709$

- Cleave H, Timmons H (1952) An Additional New Species of the Acanthocephalan Genus Neoechinorhynchus. The Journal of Parasitology 38 (1): 53. DOI: $10.2307 / 3274172$

- Crofton H (1971) A quantitative approach to parasitism. Parasitology 62: 179-193. DOI: $10.1017 /$ S0031182000071420

- Crompton D, Keymer A, Arnold S (1984) Investigating over-dispersion; Moniliformis (Acanthocephala) and rats. Parasitology 88 (2): 317-331.

- Delignette-Muller M, Pouillot R, Denis J, Dutang C (2013) fitdistrplus: help to fit of a parametric distribution to non-censored or censored data. 1.0-1. URL: http://riskassessment.r-forge.rproject.org

- Dietz K (1982) Overall population patterns in the transmission cycle of infectious agents. In: Anderson R, May R (Ed.) Population Biology of Infectious Diseases. Springer, Berlin. DOI: 10.1007/978-3-642-68635-1_6

- Dobson A (1985) The population dynamics of competition between parasites. Parasitology 91: 317-347. DOI: 10.1017/S0031182000057401

- Dorucu M, Adams C, Huntingford F, Crompton D (1995a) How fish-helminth associations arise: an example from Arctic charr in Loch Rannoch. Journal of Fish Biology 47 (6): 1038-1043. DOI: 10.1111/jfb.1995.47.issue-6

- Dorucu M, Crompton D, Huntingford F, Walters D (1995b) The ecology of endoparasitic helminth infections of brown trout (Salmo trutta) and rainbow trout (Oncorhynchus mykiss) in Scotand. Folia Parasitologica 42 (1): 29.

- Eklund A (2012) beeswarm: The bee swarm plot, an alternative to stripchart. 0.1.5. URL: http:// CRAN.R-project.org/package=beeswarm

- Golvan Y (1994) Nomenclature of the Acanthocephala. Research and Reviews in Parasitology 54: 135-205.

- Grenfell B, Smith G, Anderson R (1986) Maximum-likelihood estimates of the mortality and migration rates of the infective larvae of Ostertagia ostertagi and Cooperia oncophora. Parasitology 92: 643-652. DOI: 10.1017/S0031182000065501

- Guyatt B, Bundy D (1991) Estimating prevalence of community morbidity due to intestinal helminths: prevalence of infection as an indicator of the prevalence of disease. Transactions of the Royal Society of Tropical Medicine and Hygiene 85: 778-782. DOI: $10.1016 / 0035-9203$ (91)90453-6

- Hine P, Kennedy C (1974) The population biology of the acanthocephalan Pomphorhynchus laevis (Muller) in the River Avon. Journal of Fish Biology 6: 665-679. DOI: 10.1111/ j.1095-8649.1974.tb05108.x

- Hoffman J (1954) L'acanthocéphalose des truites de la Syre [Quelques contributions à l'étude des spécificités de l'Echinorhynchus truttae Schrank (Lühe 1911)]. Archvs Inst. gr.-d. Luxemb. sect. sc. nat. phys. math. 21: 81-98. [In French].

- Holland C, Kennedy C (1997) A checklist of parasitic helminth and crustacean species recorded in freshwater fish from Ireland. Biology and Environment 97 (3): 225-243. 
- Huffman D, Bullock W (1975) Meristograms: Graphical Analysis of Serial Variation of Proboscis Hooks of Echinorhynchus (Acanthocephala). Systematic Zoology 24 (3): 333-345. DOI: $10.2307 / 2412719$

- Huffman D, Kliever R (1977) Echinorhynchus canyonensis sp. n. (Acanthocephala) from Maynea californica (Osteichthyes: Zoarcidae) from the Monterey Submarine Canyon, California. Proceedings of the Helminthological Society of Washington 44 (2): 171.

- Lühe M (1911) Acanthocephalen. In: Brauer A (Eds) Die Süsswasserfauna Deutschlands. 16. Verlag von Gustav Fishcher, Jena, 60 pp.

- Lynch J (1936) New species of Neoechinorhynchus from the western sucker, Catostomus macrocheilus Girard. Transactions of the American Microscopical Society 55: 21-43. DOI: 10.2307/3223008

- Martínez-Aquino A, Reyna-Fabián M, Rosas-Valdez R, Razo-Mendivil U, León G, García-Varela M (2009) Detecting a Complex of Cryptic Species within Neoechinorhynchus golvani (Acanthocephala: Neoechinorhynchidae) Inferred from ITSs and LSU rDNA Gene Sequences. Journal of Parasitology 95 (5): 1040-1047. DOI: 10.1645/GE-1926.1

- Meyer A (1933) Acanthocephala. Klassen and Ordnungen des Tierreichs. 4. Akademische Verlagsgesellschaft MBH, Leipzig, $332 \mathrm{pp}$.

- Nagasawa K, Urawa S, Awakura T (1997) A checklist and bibliography of parasites of salmonids of Japan. Scientific Reports - Hokkaido Salmon Hatchery 1: 41.

- Pacala S, Dobson A (1988) The relation between the number of parasites/host and host age: population dynamics causes and maximum likelihood estimation. Parasitology 96: 197-210. DOI: 10.1017/S0031182000081762

- Pennycuick L (1971) Frequency distributions of parasites in a population of three-spined sticklebacks, Gasterosteus aculeatus L., with particular reference to the negative binomial distribution. Parasitology 63: 389-406. URL: 10.1017/S0031182000079920

- Petrochenko V (1956) Acanthocephala of Domestic and Wild Animals, volume 1. Izdatel'stvo Akademii Nauk SSSR, Moscow, 435 pp.

- Pinkster S (1978) Amphipoda. In: Illies J (Eds) Limnofauna Europaea. Gustav Fischer Verlag, Stuttgart.

- Prychitko S, Nero R (1983) Occurrence of the acanthocephalan Echinorhynchus leidyi (Van Cleave, 1924) in Mysis relicta. Canadian Journal of Zoology 61: 460-462. DOI: 10.1139/z83-061

- R Core Team (2012) R: A Language and Environment for Statistical Computing. 2.15.2. R Foundation for Statistical Computing. Release date: 201210 26. URL: http://www.R-project.org/

- Reiczigel J (2003) Confidence intervals for the binomial parameter: some new considerations. Statistics in Medicine 22 (4): 611-621. DOI: 10.1002/sim.1320

- Rózsa L, Reiczigel J, Majoros G (2000) QUANTIFYING PARASITES IN SAMPLES OF HOSTS. Journal of Parasitology 86 (2): 228-232. DOI: 10.1645/0022-3395(2000)086

[0228:QPISOH]2.0.CO;2

- Shaw D, Grenfell B, Dobson A (1998) Patterns of macroparasite aggregation in wildlife host populations. Parasitology 117: 597-610. DOI: 10.1017/S0031182098003448

- Shostak A, Dick T, Szalai A, Bernier L (1986) Morphological variability in Echinorhynchus gadi, E. leidyi and E. salmonis (Acanthocephala: Echinorhynchidae) from fishes in northern Canadian waters. Canadian Journal of Zoology 64: 985-995. DOI: 10.1139/z86-148

- Steinauer M, Nickol B, Ortí G (2007) Cryptic speciation and patterns of phenotypic variation of a highly variable acanthocephalan parasite. Molecular Ecology 16 (19): 4097-4109. DOI: $10.1111 /$ j.1365-294X.2007.03462.x

- Stoddart R, Crompton D, Walters D (1991) Influence of host strain and helminth isolate on the first phase of the relationship between rats and Moniliformis moniliformis (Acanthocephala). Journal of Parasitology 77 (3): 372. DOI: 10.2307/3283122 
- Timola O (1980) Seasonal and size-bound changes in infestation of the smelt, Osmerus eperlanus (L.), by certain parasites in the northeastern Bothnian Bay. Bothnian Bay Reports 2: 27-34.

- Väinölä R (1986) Sibling species and phylogenetic relationships of Mysis relicta (Crustacea; Mysidacea). Annals Zoologici Fennici 23: 207-221.

- Väinölä R, Valtonen E, Gibson D (1994) Molecular systematics in the acanthocephalan genus Echinorhynchus (sensu lato) in northern Europe. Parasitology 108 (1): 105. DOI: $10.1017 /$ S0031182000078574

- Väinölä R, Riddoch B, Ward R, Jones R (1994) Genetic Zoogeography of the Mysis relicta Species Group (Crustacea: Mysidacea) in Northern Europe and North America. Canadian Journal of Fisheries and Aquatic Sciences 51 (7): 1490-1505. DOI: 10.1139/f94-149

- Valtonen E (1980) Metechinorhynchus salmonis infection in the river-spawning whitefish of the Bothnian Bay. Journal of Fish Biology 17: 1-8. DOI: 10.1111/j.1095-8649.1980.tb02736.x

- Valtonen E (1983) On the ecology of acanthocephalans in the north-eastern gulf of Bothnia: dispersion and frequency distributions. In: NA (Eds) NA. 1st International Symposium of Ichthyoparasitology "Parasites and parasitic diseases of fish", Ceske Budejovice, August 8-13, 1983. $116 \mathrm{pp}$.

- Valtonen E, Crompton D (1990) Acanthocephala in fish from the Bothnian Bay, Finland. Journal of Zoology 220 (4): 619-639. DOI: 10.1111/izo.1990.220.issue-4

- Valtonen E, van Maren M, Timola O (1983) A note on the intermediate hosts of Echinorhynchus gadi Zoega in Müller (Acanthocephala) in the Baltic Sea. Aquilo Ser Zool 22: 93-97.

- Wayland M (2002) Studies on the biosystematics of species of the genus Echinorhynchus (Acanthocephala). PhD Thesis, University of Stirling, Stirling, Scotland, UK, 343 pp.

- Wayland M (2010) Proboscis profiler: a tool for detecting acanthocephalan morphotypes. Systematic Parasitology 76 (3): 159-167. DOI: 10.1007/s11230-010-9245-z

- Wayland M, Gibson D, Sommerville C (2005) Morphometric discrimination of two allozymically diagnosed sibling species of the Echinorhynchus gadi Zoega in Müller complex (Acanthocephala) in the North Sea. Systematic Parasitology 60 (2): 139-149. DOI: 10.1007/s11230-004-1388-3

- Wolff R (1984) Mysis relicta as intermediate host of an acanthocephalan parasite. Transactions of the Illinois Academy of Science 77: 1-2.

- Zdzitowiecki K, Valtonen E (1987) Description of Echinorhynchus bothniensis sp. $n$. (Acanthocephala), a parasite of smelt Osmerus eperlanus L. in Bothnian Bay. Acta Parasitologica Polonica 32 (3): 233-238.

- Zhukov E (1960) Endoparasitic worms of fish from the Sea of Japan and the shallow waters of the South Kuril Islands. Trudy Zoologischekago Inst Leningrad 28: 3-129.

\section{Supplementary materials}

\section{Suppl. material 1: Standard morphometric and meristic data from females.}

Authors: Matthew T Wayland

Data type: morphological and meristic

Brief description: Comma separated value (csv) file of morphometric data from females. Rows are specimens and columns (column three onwards) are morphometric variables (e.g. proboscis length) or meristic variables (e.g. number of longitudinal rows of hooks). All morphometric measurements are in micrometres. The first column is species and the second column is a unique identifier for the specimen. The unique identifier is composed of two parts: the part before the full stop indicates the sample (please see table 1); the number after the full stop indicates the 
specimen number. In the species column, E. bothniensis and E. 'bothniensis' are listed as bothniensis 1 and bothniensis2, respectively.

Filename: female_morphometrics.csv - Download file $(16.65 \mathrm{~kb})$

\section{Suppl. material 2: Standard morphometric and meristic data from males.}

Authors: Matthew T Wayland

Data type: morphological and meristic

Brief description: Comma separated value (csv) file of morphometric data from males. Rows are specimens and columns (column three onwards) are morphometric variables (e.g. proboscis length) or meristic variables (e.g. number of longitudinal rows of hooks). All morphometric measurements are in micrometres. The first column is species and the second column is a unique identifier for the specimen. The unique identifier is composed of two parts: the part before the full stop indicates the sample (please see table 1); the number after the full stop indicates the specimen number. In the species column E. bothniensis and E. 'bothniensis' are listed as bothniensis1 and bothniensis2, respectively. Notation for cement gland pattern from Shostak et al. (1986): B, clumped, three staggered pairs; C, chainlike, two pairs and two singles; D, chainlike, one pair and four singles; E, chainlike, six singles.

Filename: male_morphometrics.csv - Download file (12.64 kb)

\section{Suppl. material 3: Egg and acanthor dimensions}

Authors: Matthew T Wayland

Data type: morphological

Brief description: Comma separated value file with 6 columns: species, specimen, egg length, acanthor length, egg width, acanthor width. All measurements in micrometres. The unique identifier for specimen is composed of two parts: the part before the full stop indicates the sample (please see table 1); the number after the full stop indicates the specimen number. Three eggs were measured from each gravid female. In the species column $E$. bothniensis and $E$. 'bothniensis' are listed as bothniensis1 and bothniensis2, respectively.

Filename: eggs.csv - Download file $(6.21 \mathrm{~kb})$

\section{Suppl. material 4: Hook measurement data from females}

Authors: Matthew T Wayland

Data type: morphological

Brief description: The file is a comma separated value (CSV) format suitable for input to the Acanthocephalan Proboscis Profiler software (http://acanthocephala.sourceforge.net). It includes data from one of the paratypes of E. bothniensis from the Bothnian Bay, Baltic Sea (specimen: b1.01).

The file has 5 columns: specimen, group, hook, length and base.

specimen - unique identifier for the specimen group - name of group ( $E$. bothniensis and $E$. 'bothniensis' are listed as bothniensis1 and bothniensis2, respectively) hook - numerical position 
of hook in longitudinal row as counted from the distal end of the probocis length - length of hook blade (micrometres) base - width of hook base (micrometres)

Filename: female_hook_measurements.csv - Download file $(22.38 \mathrm{~kb})$

\section{Suppl. material 5: Hook measurement data from males}

Authors: Matthew T Wayland

Data type: morphological

Brief description: The file is a comma separated value (CSV) format suitable for input to the Acanthocephalan Proboscis Profiler software (http://acanthocephala.sourceforge.net).

The file has 5 columns: specimen, group, hook, length and base.

specimen - unique identifier for the specimen

group - name of group (following convention used in other data files, E. bothniensis is listed as bothniensis1)

hook - numerical position of hook in longitudinal row as counted from the distal end of the probocis

length - length of hook blade (micrometres)

base - width of hook base (micrometres)

Filename: male_hook_measurements.csv - Download file (15.41 kb)

\section{Suppl. material 6: Frequency distribution of Echinorhynchus truttae in Salmo trutta from Loch Coulter Burn}

Authors: Matthew T Wayland

Data type: ecological

Brief description: Comma-separated value (CSV) file with two columns: host fork length $(\mathrm{mm})$ and number of worms. Host fish were sampled from Loch Coulter Burn (National Grid Reference NS 761 865) on 20/9/1996. Acanthocephalan voucher specimens: BM(NH) 2002.2.4.276-283.

Filename: truttae_Loch_Coulter_host_distribution.csv - Download file (278.00 bytes)

\section{Suppl. material 7: Frequency distribution of Echinorhynchus truttae in Salmo trutta from Loch Walton Burn}

Authors: Matthew T Wayland

Data type: ecological

Brief description: Comma-separated value (CSV) file with two columns: host fork length $(\mathrm{mm})$ and number of worms. Host fish were sampled from Loch Walton Burn (National Grid Reference NS 668 865) on 24/6/1996. Acanthocephalan voucher specimens: BM(NH) 2002.2.4.264-275.

Filename: truttae_Loch_Walton_host_distribution.csv - Download file (278.00 bytes) 
Suppl. material 8: Boxplots showing sexual dimorphism in morphometric and meristic characters in Echinorhyhus truttae

Authors: Matthew T Wayland

Data type: morphological

Brief description: Boxplots showing sexual dimorphism in morphometric and meristic data for Echinorhynchus truttae. For numbers specimens in each plot please see tables 2 and 3 .

Filename: truttae_sexual_dimorphism_boxplots.pdf - Download file $(8.43 \mathrm{~kb})$

Suppl. material 9: Boxplots of morphometric and meristic data from female acanthocephalans.

Authors: Matthew T Wayland

Data type: morphological

Brief description: Boxplots of morphometric and meristic data from female E. bothniensis (Lake Keitele), E. 'bothniensis' and E. truttae.

Filename: female_morphometric_boxplots.pdf - Download file $(12.20 \mathrm{~kb})$

\section{Suppl. material 10: Boxplots of morphometric and meristic data from male acanthocephalans.}

Authors: Matthew T Wayland

Data type: morphological

Brief description: Boxplots of morphometric and meristic data from male E. bothniensis (Lake Keitele), E. 'bothniensis' and E. truttae.

Filename: male_morphometric_boxplots.pdf - Download file (13.01 kb) 